\title{
ARTICLE DL-3-n-butylphthalide ameliorates diabetes-associated cognitive decline by enhancing PI3K/Akt signaling and suppressing oxidative stress
}

Bei-ni Wang ${ }^{1,2}$, Cheng-biao $\mathrm{Wu}^{3}$, Zi-miao Chen ${ }^{1}$, Pei-pei Zheng ${ }^{2}$, Ya-qian Liu ${ }^{2}$, Jun Xiong ${ }^{2}$, Jing-yu Xu ${ }^{4}$, Pei-feng Li ${ }^{1}$, Abdullah Al Mamun ${ }^{2}$, Li-bing $\mathrm{Ye}^{2}$, Zhi-long Zheng ${ }^{2}$, Yan-qing $\mathrm{Wu}^{4}$, Jian $\mathrm{Xiao}^{1,2}$ and Jian Wang ${ }^{1}$

DL-3-n-Butylphthalide (DL-NBP), a small molecular compound extracted from the seeds of Apium graveolens Linn (Chinese celery), has been shown to exert neuroprotective effects due to its anti-inflammatory, anti-oxidative and anti-apoptotic activities. DL-NBP not only protects against ischemic cerebral injury, but also ameliorates vascular cognitive impairment in dementia patients including AD and PD. In the current study, we investigated whether and how DL-NBP exerted a neuroprotective effect against diabetes-associated cognitive decline (DACD) in $d b / d b$ mice, a model of type- 2 diabetes. $d b / d b$ mice were orally administered $\operatorname{DL}-\operatorname{NBP}\left(20,60,120 \mathrm{mg} \cdot \mathrm{kg}^{-1} \cdot \mathrm{d}^{-1}\right)$ for 8 weeks. Then the mice were subjected to behavioral test, their brain tissue was collected for morphological and biochemical analyses. We showed that oral administration of DL-NBP significantly ameliorated the cognitive decline with improved learning and memory function in Morris water maze testing. Furthermore, DL-NBP administration attenuated diabetes-induced morphological alterations and increased neuronal survival and restored the levels of synaptic protein PSD95, synaptophysin and synapsin-1 as well as dendritic density in the hippocampus, especially at a dose of $60 \mathrm{mg} / \mathrm{kg}$. Moreover, we revealed that DL-NBP administration suppressed oxidative stress by upregulating Nrf2/HO-1 signaling, and increased brain-derived neurotrophic factor (BDNF) expression by activating PI3K/Akt/CREB signaling in the hippocampus. These beneficial effects of DL-NBP were observed in high glucose-treated PC12 cells. Our results suggest that DL-NBP may be a potential pharmacologic agent for the treatment of DACD.

Keywords: diabetes-associated cognitive decline; DL-3-n-butylphthalide; cognitive function; hippocampus; BDNF; oxidative stress; neuroprotection

Acta Pharmacologica Sinica (2021) 42:347-360; https://doi.org/10.1038/s41401-020-00583-3

\section{INTRODUCTION}

Type-2 diabetes (T2D) is a serious chronic metabolic disorder, which adversely affects multiple organs due to its long-term complications, including the brain. As early as 1922, it was reported that diabetes caused cognitive dysfunction [1]. Numerous clinical and epidemiological studies have also demonstrated that diabetes is closely related to dementia [2, 3]. Moreover, longitudinal studies have demonstrated that the rate of cognitive decline in patients with T2D is up to two times faster than in nondiabetic persons [4]. These findings highlight the pathogenic role of diabetes in cognitive impairment. Notwithstanding, the exact molecular mechanisms and therapeutic strategies needed to tackle diabetes-associated cognitive decline (DACD) are still unclear.

Multiple studies have demonstrated that oxidative stress underlies diabetes development and its related complications $[5,6]$. An imbalance between oxidative stress and antioxidant capacity in the brain can eventually lead to cell injury, including mitochondrial damage and neuronal apoptosis. A prior study demonstrated that oxidative stress may be associated with learning and memory deficits [7]. Increased oxidative damage and decreased antioxidant enzymatic activity localize in the synaptic regions of the brain in Alzheimer's disease (AD) patients [8]. On the other hand, hippocampal synaptic plasticity has been proposed as the mechanism underlying spatial learning and memory [9]. Therefore, it is reasonable to speculate that enhancing antioxidant defenses, for example by administering antioxidants, may reduce damage caused by oxidative stress, ameliorating synaptic dysfunction and neuronal injury in DACD [10].

Nuclear factor erythroid 2-related factor 2 (Nrf2) is a member of the basic leucine zipper family of transcription factors [11]. It is a master regulator during oxidative stress, controlling antioxidant gene expression and increasing the production of antioxidant

\footnotetext{
${ }^{1}$ Department of Hand Surgery and Peripheral Neurosurgery, The First Affiliated Hospital of Wenzhou Medical University, Wenzhou 325000, China; ${ }^{2}$ School of Pharmaceutical Science, Wenzhou Medical University, Wenzhou 325000, China; ${ }^{3}$ Research Center, Affiliated Xiangshan Hospital, Wenzhou Medical University, Ningbo 315700 , China and ${ }^{4}$ The

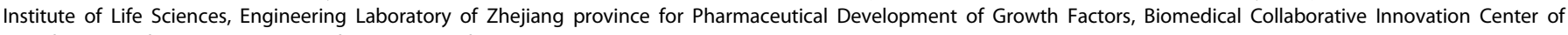
Wenzhou, Wenzhou University, Wenzhou 325035, China

Correspondence: Yan-qing Wu (yqwu220946@yeah.net) or Jian Xiao (xfxj2000@126.com) or Jian Wang (jianwang0516@126.com)

These authors contributed equally: Bei-ni Wang, Cheng-biao Wu, Zi-miao Chen
}

Received: 1 August 2020 Accepted: 16 November 2020

Published online: 18 January 2021 
enzymes, such as heme oxygenase 1 (HO-1) [12]. Nrf2 plays a significant role in cellular adaptive responses to oxidative stress. The Nrf2/HO-1 pathway was recently shown to have a significant neuroprotective role in epilepsy [13, 14]. In addition, studies have reported that oxidative stress and damage are increased in aging mammals, and that Nrf2 knockout mice exhibited decreased expression of Nrf2 and increased susceptibility to oxidative damage, indicating that Nrf2 has neuroprotective effects [15]. The phosphatidylinositol-3-kinase (PI3K)/Akt pathway is a key regulator of cell survival and proliferation that is widely expressed in the central nervous system. It is a well-known pathway that is involved in the promotion of neuronal survival $[16,17]$ Studies have reported that activated nuclear Nrf2 regulates several endogenous redox-regulated enzymes, such as $\mathrm{HO}-1$ and glutathione cysteine ligase modulatory subunit, via phosphorylated PI3K and phosphorylated AKT [18-20]. Based on these findings, we hypothesized that PI3K-Nrf2 crosstalk was a key factor linked to DACD.

The CAMP response element-binding protein (CREB) family of leucine zipper transcription factors is involved in regulating longterm synaptic plasticity and memory-related gene expression, including that of brain-derived neurotrophic factor (BDNF) [21]. In addition to its classical trophic activity, BDNF effectively regulates synaptic plasticity and promotes learning and memory [22, 23]. CREB activity and BDNF expression are remarkably suppressed in $A D$ patients and $A D$ animal models with associated cognitive decline [24, 25]. Previous studies have also shown that diabetes results in the downregulation of BDNF expression in the cortex or hippocampus $[26,27]$. Protein kinase $B(A k t)$ is one of the major kinases regulating CREB activity. Inhibition of Akt phosphorylation reduces CREB activity, regulating neuronal survival and synaptic function in $A D$ and Parkinson's disease (PD) $[3,28]$. Therefore, evidence suggests that Akt/CREB signaling may be one of the key molecular mechanisms underlying DACD development.

DL-3-n-butylphthalide (DL-NBP), a small molecular compound extracted from the seeds of Apium graveolens Linn (Chinese celery), was approved for the treatment of ischemic stroke by the State Food and Drug Administration of China in 2002 [29]. Numerous studies have explored its neuroprotective effects and possible mechanisms of action. DL-NBP not only protects against ischemic cerebral injury, but also ameliorates vascular cognitive impairment in dementia patients, including AD and PD [30-32]. Although the specific pharmacologic targets of DL-NBP are unknown, cumulative studies indicate that its neuroprotective effects depend on multiple antioxidant, anti-apoptotic, antiinflammatory, and pro-neurogenesis properties [33-35]. Therefore, these findings suggest that DL-NBP could be a potential multifunctional-candidate drug for the treatment of several neurodegenerative diseases, including DACD.

The aim of this study was to investigate whether DL-NBP had a neuroprotective effect against DACD in $d b / d b$ mice, facilitating functional recovery, and to explore the related molecular mechanisms. Our results indicate that DL-NBP treatment significantly reduces neuronal apoptosis, improves synaptic dysfunction and ameliorates cognitive decline in $d b / d b$ mice. This beneficial effect is most likely mediated by enhanced PI3K/Akt/CREB signaling and reduced oxidative stress mediated through the $\mathrm{PI3K} / \mathrm{Akt} / \mathrm{Nrf2}$ signaling pathway. Collectively, these findings help clarify the therapeutic effects of DL-NBP treatment in DACD and the underlying mechanisms of action.

\section{MATERIALS AND METHODS}

Animals and experimental design

Male $d b / d b$ mice (12-week-old, C57BLKS/J-leprdb/leprdb) and their non-diabetic $d b / m$ littermates were purchased from the Animal Research Center of Nanjing University (Nanjing, China). And $d b / m$ litter mates are the heterozygous mouse from the same litter of $d b / d b$ mice. All experimental animal procedures were approved by the Ethics Committee of Wenzhou Medical University and were performed in accordance with the Guidance for the Care and Use of Laboratory Animals. Animals were group housed at $22^{\circ} \mathrm{C}$ under a $12 \mathrm{~h}$ light $/ 12 \mathrm{~h}$ dark cycle, provided with water and fed a standard diet. DL-NBP (purity $>98 \%$ ) was supplied by CSPC, the Institute of Pharmaceutical Research (Shijiazhuang, China). It was diluted in vegetable oil, as previously reported $[9,36]$. The $d b / d b$ mice were randomly divided into four groups: $d b / d b$ mice, $d b / d b+$ LDL-NBP ( $20 \mathrm{mg} / \mathrm{kg}$ body weight), $d b / d b+$ MDL-NBP (60 mg/kg body weight), $d b / d b+$ HDL-NBP $(120 \mathrm{mg} / \mathrm{kg}$ body weight) (Table 1). DL-NBP was administered by oral gavage and mice were treated once daily for 8 weeks. The $d b / m$ mice and $d b / d b$ mice received vehicle only, consisting of vegetable oil. Metformin $(200 \mathrm{mg} / \mathrm{kg})$, a classical anti-diabetic drug, was administered to $d b / d b$ mice as positive control group for 8 weeks. After 8 weeks, mice were subjected to the Morris water maze test. After the behavioral test, animals were anesthetized with $10 \%$ chloral hydrate $(3.5 \mathrm{~mL} / \mathrm{kg})$ and perfused initially with $0.9 \%$ saline solution via cardiac puncture. For immunofluorescence,

Table 1. The sample pet group in every experiment.

\begin{tabular}{|c|c|c|c|c|c|c|c|}
\hline Methods & Morris water maze test & H\&E staining & Nissl staining & Western blot & DHE staining & MDA content assay & IF staining \\
\hline \multicolumn{8}{|c|}{ Experiment 1 (Sample size) } \\
\hline$d b / m$ & 12 & 5 & 5 & 3 & / & / & / \\
\hline$d b / d b+\mathrm{LDL}-\mathrm{NBP}$ & 12 & 5 & 5 & 3 & / & / & / \\
\hline$d b / d b+\mathrm{MDL}-\mathrm{NBP}$ & 12 & 5 & 5 & 3 & l & / & / \\
\hline$d b / m$ & / & / & / & 3 & 5 & 4 & $\geq 3$ \\
\hline$d b / d b$ & / & / & / & 3 & 5 & 4 & $\geq 3$ \\
\hline$d b / d b+\mathrm{MDL}-\mathrm{NBP}$ & / & / & I & 3 & 5 & 4 & $\geq 3$ \\
\hline \multicolumn{8}{|c|}{ Experiment 3 (Sample size) } \\
\hline$d b / m$ & 6 & / & I & / & I & / & / \\
\hline
\end{tabular}


hematoxylin \& eosin (H\&E) and Nissl staining, animals were perfused with $4 \%$ paraformaldehyde (PFA) in $0.1 \mathrm{M}$ PBS following the initial saline solution perfusion. Next, the brain tissues were rapidly removed and post-fixed by immersion in 4\% PFA for $24 \mathrm{~h}$. For Western blot analysis, the whole hippocampus was removed and snap frozen at $-80^{\circ} \mathrm{C}$.

\section{Morris water maze (MWM) testing}

All mice were trained and tested in the MWM to monitor their spatial learning and memory abilities as previously described $[37,38]$. The test was performed in a circular pool with a diameter of $120 \mathrm{~cm}$ and a height of $40 \mathrm{~cm}$ (Jiliang; Shanghai, China). It was filled with opaque water colored with milk powder and maintained at $26 \pm 1{ }^{\circ} \mathrm{C}$. Using a hidden circular platform, the training was carried out in six blocks, each consisting of three $60 \mathrm{~s}$ trials, separated by $20 \mathrm{~min}$ inter-block intervals. During the training period, the platform remained in the same location relative to the distal cues in the room. In each trial, mice were placed in the water at different starting locations $(E, S, W$, and $N$ ), equally spaced from each other, and diverging from the goal location by $45^{\circ}$. About $1 \mathrm{~h}$ after the sixth block, the hidden platform was removed, and the mice were scored during a $60 \mathrm{~s}$ probe trial. They were scored for latency to reach the goal and for memory recall, which was determined by the number of crossings over the previous platform location. Another probe trial was performed $24 \mathrm{~h}$ after training to assess memory consolidation and memory retrieval.

\section{Hematoxylin \& Eosin (H\&E) and Nissl staining}

The brain tissues were carefully collected and fixed in 4\% PFA solution for $24 \mathrm{~h}$. Next, they were dehydrated in alcohol and embedded in paraffin. After that, $5 \mu \mathrm{m}$ brain tissue sections were dewaxed, hydrated, and stained with hematoxylin and eosin (H\&E) solution (Beyotime, China). For Nissl staining, the slides were stained with cresol violet and Nissl differentiation solution, according to the manufacturer's instructions (Beyotime, China). Finally, the slides were observed under a light microscope (Nikon, Japan).

PC12 cell culture and treatments

PC12 cells are commonly used as an in vitro model to study central nervous system neurons [39-41]. PC12 cells were purchased from the Cell Storage Center of Wuhan University (Wuhan, China) and were cultured in Dulbecco's modified Eagle's medium (DMEM, Invitrogen, Carlsbad, CA, USA) supplemented with $10 \%$ fetal bovine serum (FBS, Invitrogen, Carlsbad, CA, USA) and antibiotics (100 units $/ \mathrm{mL}$ penicillin, $100 \mu \mathrm{g} / \mathrm{mL}$ streptomycin). PC12 cells were incubated at $37^{\circ} \mathrm{C}$ in a humidified atmosphere containing $5 \% \mathrm{CO}_{2}$. A $10 \mathrm{mM}$ stock solution of DL-NBP was prepared in DMSO. To establish a high glucose cell model in vitro, $30 \mathrm{mM}$ glucose was added to the medium with/without DL-NBP $(10 \mu \mathrm{M})$. Cells were exposed to this HG condition for $24 \mathrm{~h}$. HG plus DL-NBP was the HG + DL-NBP group. Cells incubated in HG medium only were the HG group. Control cells were cultured in normal medium. To evaluate the effects of Nrf2 signaling on high glucose treated-PC12 cells, cells were incubated for $24 \mathrm{~h}$ with both DL-NBP and the Nrf2 inhibitor ML385 (5 $\mu \mathrm{M}$, MedChem Express, USA) [42]. To evaluate the effects of PI3K/Akt signaling on high glucose treated-PC12 cells, cells were incubated for $2 \mathrm{~h}$ with the PI3K inhibitor LY294002 (LY, $20 \mu \mathrm{M}$, Abcam, Cambridge, MA, USA) before adding DL-NBP [43]. Thus, there were four experimental groups: Control (CON), HG, HG + DL-NBP, HG + DL-NBP + ML385 and HG + DL-NBP + LY294002.

\section{Intracellular-ROS generation}

Intracellular-ROS generation was measured using the ROS Assay Kit (DCFH-DA, S0033, Beyotime, China). PC12 cells were plated in 6 -well plates at a density of $1 \times 10^{5}$ cells $/ \mathrm{mL}$ and incubated for
$24 \mathrm{~h}$. After treatment with HG, cells were incubated with and without DL-NBP for $24 \mathrm{~h}$. Next, $10 \mu \mathrm{M} \mathrm{2}$,', $7^{\prime}$-dichlorodihydrofluorescein diacetate (DCFH-DA) was added to the culture medium and cells were incubated for $30 \mathrm{~min}$. Nuclei were labeled with DAPI and fluorescence was detected with a confocal laser microscope (Nikon, Tokyo, Japan).

TUNEL staining

TUNEL staining was performed using an In Situ Cell Death Detection Kit (Beyotime, China). Following the standard protocol, fixed cell slides were incubated in $0.3 \%$ Triton X-100 for 5 min at room temperature. The slides were washed three times with PBS, followed by incubation in the TUNEL reaction mixture for $1 \mathrm{~h}$ at $37^{\circ} \mathrm{C}$. After washing with PBS three more times ( $5 \mathrm{~min} /$ wash), DAPI was added to the slides. Cells were imaged using a confocal laser microscope (Nikon, Tokyo, Japan).

\section{Western blot analysis}

Hippocampal tissue and PC12 cells were collected and lysed using ice-cold RIPA buffer containing a protease inhibitor cocktail $(10 \mu \mathrm{g} /$ $\mathrm{mL}$, GE Healthcare Biosciences, PA, USA). Following centrifugation at $12,000 \mathrm{rpm}$ for $20 \mathrm{~min}$ at $4{ }^{\circ} \mathrm{C}$, protein concentration in the supernatant was quantified with BCA reagent (Thermo, Rockford, IL, USA). For Nrf2 detection, PC12 cells were collected in PBS buffer. Next, cytosolic and nuclear proteins were extracted using a nuclear and cytoplasmic protein extraction kit (Beyotime, China), followed by centrifugation at $12,000 \mathrm{rpm}$ for $10 \mathrm{~min}$ at $4{ }^{\circ} \mathrm{C}$ to separate the nuclear components [44]. The protein samples were separated using $10 \%$ or $12 \%$ gels and transferred to polyvinylidene difluoride membranes (Bio-Rad, Hercules, CA, USA). Membranes were blocked with $5 \%$ non-fat dry milk in TBST solution for $2 \mathrm{~h}$ at room temperature. After blocking, the membranes were washed three times with PBS at room temperature. Next, the membranes were incubated overnight at $4{ }^{\circ} \mathrm{C}$ with the following specific primary antibodies: PSD95 (1:1000, Abcam), Synaptophysin (1:1000, Abcam), Synapsin-1 (1:1000, CST), NOX2 (1:1000, Abcam), NOX4 (1:1000, Abcam), Nrf2 (1:1000, Abcam), HO-1 (1:1000, Proteintech, China), NQO1 (1:1000, Abcam), SOD2 (1:2000, Proteintech), CREB (1:1000, CST), p-CREB (S133) (1:1000, CST, Danvers, MA, USA), Akt (1:1000, CST), p-Akt (1:1000, CST), BDNF (1:1000, Abcam), Bcl-2 (1:2000, Affinity, China), Bax (1:1000, Proteintech), Cleaved-caspase3 (1:1000, Affinity) and GAPDH (1:10000, Bioworld, China). After washing three times with TBST, the membranes were incubated $2 \mathrm{~h}$ at room temperature with horseradish peroxidase-conjugated secondary antibodies (1:10000, Proteintech). The protein bands were visualized using the ChemiDocXRS+Imaging System (BioRad). Finally, the intensity of the bands was quantified using Image Lab 3.0 software (Bio-Rad).

\section{Immunofluorescence staining}

After dewaxing and hydration, brain tissue sections were incubated in $3 \% \mathrm{H}_{2} \mathrm{O}_{2}$ for $15 \mathrm{~min}$, then in $80 \%$ alcohol for 30 min, and then washed three times with PBS ( 5 min per wash). Next, slides were blocked with $5 \%$ bovine serum albumin (BSA) at $37^{\circ} \mathrm{C}$ for $30 \mathrm{~min}$. Subsequently, the tissue sections were incubated overnight at $4{ }^{\circ} \mathrm{C}$ with primary antibodies specific for BDNF (1:600, Abcam), SMI-32(1:500, Abcam) and Cleaved-caspase-3 (1:1000, CST). After overnight incubation, the slides were washed three times with PBS solution and incubated for $2 \mathrm{~h}$ at $37^{\circ} \mathrm{C}$ with specific secondary Alexa Fluor 647 or Alexa Fluor 488 (1:1000, Abcam)labeled antibodies. In the case of PC12 cells, they were washed with PBS solution, fixed in 4\% PFA for $30 \mathrm{~min}$, and blocked with $5 \%$ BSA for $30 \mathrm{~min}$ at $37^{\circ} \mathrm{C}$. Then, the cells were incubated overnight at $4{ }^{\circ} \mathrm{C}$ with primary antibodies specific for Cleavedcaspase-3 (1:1000, CST) and Nrf2 (1:500, Abcam). Alexa Fluor 488labeled secondary antibodies were added at a 1:1000 dilution for $2 \mathrm{~h}$, and nuclei were labeled with DAPI. Images were captured using a confocal laser microscope (Nikon, Tokyo, Japan). 

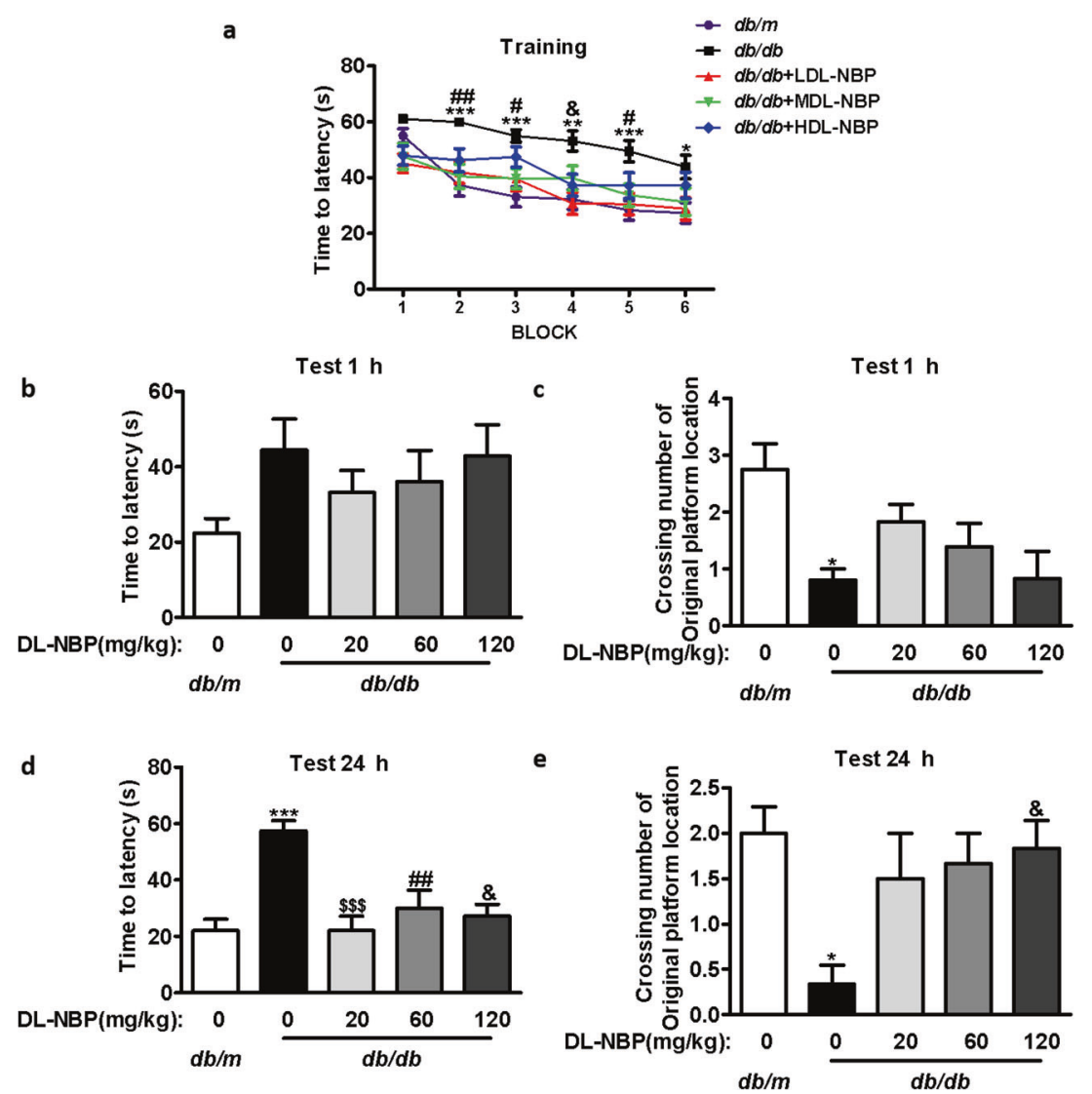

Fig. 1 DL-NBP treatment ameliorates diabetes-induced cognitive decline. a Learning curve in the Morris water maze test (divided in six blocks). b Mean latency time to find the platform during the probe trial ( $1 \mathrm{~h}$ after training). c Number of crossings over the original platform location ( $1 \mathrm{~h}$ after training). d Mean latency time to find the platform during the probe trial ( $24 \mathrm{~h}$ after training). e Number of crossings over the original platform location (24 h after training), $n=12$. ${ }^{*} P<0.05,{ }^{* *} P<0.01,{ }^{* * *} P<0.001$ indicates $d b / m$ group vs. $d b / d b$ group; ${ }^{\$ \$} P<0.001$ indicates $d b / d b+$ LDL-NBP group vs. $d b / d b$ group; ${ }^{\#} P<0.05,{ }^{\# \#} P<0.01$ indicates $d b / d b+$ MDL-NBP group vs. $d b / d b$ group; ${ }^{~} P<0.05$, indicates $d b / d b+$ HDL-NBP group vs. $d b / d b$ group.

Assessment of malondialdehyde (MDA) content and dihydroethidium (DHE) staining

To determine the index of plasma lipid peroxidation, the MDA content was assayed by using an MDA Detection Kit (Beyotime, China). Precipitated MDA reacts with thiobarbituric acid, producing a red substance with a maximum absorption peak at $535 \mathrm{~nm}$. DHE staining was performed to detect superoxide levels (Beyotime, China). DHE reacts with superoxide that is bound to cellular components, including protein and DNA, and emits a bright red fluorescence. Brain tissue sections were dewaxed, hydrated, incubated in a $3 \% \mathrm{H}_{2} \mathrm{O}_{2}$ solution for $15 \mathrm{~min}$, in $80 \%$ alcohol for $30 \mathrm{~min}$, and then washed three times with PBS. After washing, the slides were incubated for $1 \mathrm{~h}$ at $37^{\circ} \mathrm{C}$ in a blocking solution. Next, the brain tissue sections were incubated in a 1 $\mathrm{mmol} / \mathrm{L}$ DHE solution for $10 \mathrm{~min}$ at room temperature, washed three times with PBS, stained with DAPI and mounted using aqueous mounting medium (Sigma, St.Louis, MO, USA). Finally, images were captured using a confocal laser microscope (Nikon, Tokyo, Japan).

Statistical analysis

Results are expressed as the mean \pm SEM. Experiments were repeated at least three times, and the tissues for each replicate were from different mice. Statistical significance was determined by one-way analysis-of-variance (ANOVA), followed by Tukey's post hoc test. $P$ values below 0.05 were considered statistically significant.

\section{RESULTS}

DL-NBP treatment ameliorates diabetes-induced cognitive decline

We assessed the potential effect of DL-NBP treatment on DACD by means of the MWM test. As shown in Fig. 1a, the time required for mice to reach the platform decreased significantly after training. However, there was a significant difference in the latency to reach the platform among the different groups during the training period. Compared with $d b / \mathrm{m}$ mice, $d b / d b$ mice required a longer time to reach the platform between blocks 2 and 6 . DL-NBP treatment significantly decreased this latency period, especially in the LDL-NBP and MDL-NBP groups (Fig. 1a). Then we removed the platform and measured the difference in spatial memory ability of the mice at $1 \mathrm{~h}$ and $24 \mathrm{~h}$ after training. During the probe trial, we observed that $d b / d b$ mice showed longer latency periods and fewer numbers of crossings over the platform than $\mathrm{db} / \mathrm{m}$ mice. However, DL-NBP treatment significantly reversed this trend at $1 \mathrm{~h}$ (Fig. 1b, c). After $24 \mathrm{~h}$, memory retention of the platform's location in $d b / d b$ mice was reduced when compared with $d b / m$ mice (Fig. 1d, e). Importantly, DL-NBP treatment reversed this deficit (Fig. 1d, e). Furthermore, we used $d b / d b$ mice treated-metformin as a positive control group and further evaluated the role of DLNBP. During behavioral studies, we found that both DL-NBP and metformin effectively alleviated cognitive impairment in $d b / d b$ mice, but there was no significant difference in spatial learning and memory ability between DL-NBP-treated $d b / d b$ mice and the metformin-treated $d b / d b$ mice (Supplementary Fig. 1a-e). These 

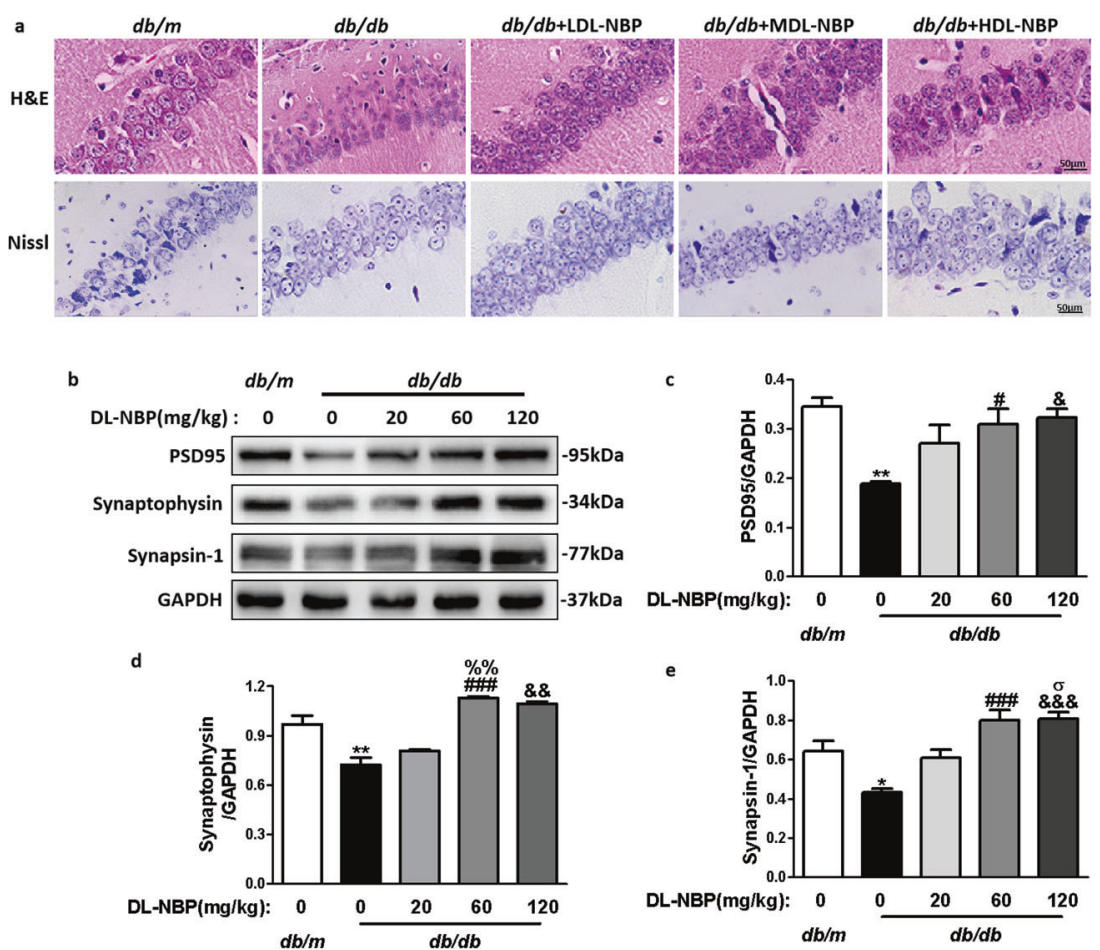

Fig. 2 DL-NBP attenuates diabetes-induced morphological alterations and synaptic dysfunction in DACD. a Representative H\&E and Niss stained sections of the CA1 region in the hippocampus. Scale bar $=50 \mu \mathrm{m}, n=5$. b Representative Western blots showing PSD95, synaptophysin and synapsin-1 expression levels. c-e Quantitative analysis of PSD95, synaptophysin and synapsin- 1 expression levels, $n=3$. ${ }^{*} P<0.05$, ${ }^{* *} P<0.01$ indicates $d b / m$ group vs. $d b / d b$ group; ${ }^{\#} P<0.05$, \#\#\# $P<0.001$ indicates $d b / d b+M D L-N B P$ group vs. $d b / d b$ group; ${ }^{\&} P<0.05$, ${ }^{\&} \&<<0.01,{ }^{\&} \& p<0.001$ indicates $d b / d b+$ HDL-NBP group vs. $d b / d b$ group; ${ }^{\%} \%<0.01$ indicates $d b / d b+$ MDL-NBP group vs. $d b / d b+$ LDL-NBP group; ${ }^{\circ} P<0.05$ indicates $d b / d b+$ HDL-NBP group vs. $d b / d b+$ LDL-NBP group.

results suggest that DL-NBP treatment ameliorates diabetesinduced learning and memory impairments.

DL-NBP treatment reduces diabetes-induced morphological changes and synaptic dysfunction

Next, we assessed whether DL-NBP treatment affected hippocampal morphology in $d b / d b$ mice. We observed that the hippocampal CA1 region in $\mathrm{db} / \mathrm{m}$ mice exhibited normal morphology, with evident nucleoli, and clear nuclear membranes (Fig. 2a). However, diabetes induced neuronal loss in the hippocampus, which looked sparsely arranged, with vague cellular boundaries. Importantly, DL-NBP treatment substantially reversed these abnormalities, especially in $d b / d b+$ MDL-NBP group (Fig. 2a). These results suggest that DL-NBP had a substantial neuroprotective effect, inhibiting DACD development, neuronal loss and changes in the structure of the hippocampus in $d b / d b$ mice.

We also evaluated the expression levels of synaptic-related proteins (PSD95, synaptophysin and synapsin-1) in the hippocampus, which constitute the molecular basis for synaptic plasticity. PSD95 (Fig. 2b, c), synaptophysin (Fig. 2b, d) and synapsin-1 (Fig. $2 b$, e) protein expression levels were notably reduced in the hippocampus of $d b / d b$ mice when compared with $d b / m$ mice. Treatment with 60 and $120 \mathrm{mg} / \mathrm{kg}$ of DL-NBP significantly upregulated the expression of these three proteins in $d b / d b$ mice, especially at $60 \mathrm{mg} / \mathrm{kg}$. However, at the low dose of DL-NBP (20 $\mathrm{mg} / \mathrm{kg}$ ), there were no significant differences between the treated and the $d b / d b$ groups. In summary, these results demonstrate that DL-NBP increases the expression levels of hippocampal synapticrelated proteins in $d b / d b$ mice. We selected a dose of $60 \mathrm{mg} / \mathrm{kg}$ of DL-NBP (MDL-NBP) to perform the subsequent experiments.

To assess the effects of DL-NBP on the density of synapse, we analyzed the dendritic density in the hippocampus of mice using SMl-32 staining [45]. As shown in Fig. 3, diabetes induced the abnormalities in the dendritic architecture with the appearance of dystrophic neuritis, reduced length and density of spine in the hippocampus (Fig. 3a-d). Dendritic spine densities and length in the hippocampus from the $60 \mathrm{mg} / \mathrm{kg}$ DL-NBP-treated group were markedly reversed when compared with those in $d b / d b$ mice (Fig. 3a-d). These results suggested that DL-NBP improves synaptic morphology, consequently promotes the recovery of neurotransmission function.

DL-NBP reduces neuronal apoptosis in the hippocampus of $d b / d b$ mice

We also measured apoptosis-related protein expression levels (Cleaved-caspase-3, Bcl-2 and Bax) in the hippocampus of $d b / d b$ mice to evaluate whether DL-NBP administration could ameliorate neuronal apoptosis during DACD development. Compared with the $d b / m$ group, $\mathrm{Bcl}-2$ (Fig. $4 \mathrm{a}$, b) protein expression levels was significantly reduced in the hippocampus of $d b / d b$ mice. In contrast, Cleaved-caspase-3 (Fig. 4a, d) and Bax (Fig. 4a, c) expression levels were significantly increased in the hippocampus of $d b / d b$ mice when compared with $d b / m$ mice. This pattern was reversed by DL-NBP treatment. Consistent with the Western blot results, the number of Cleaved-caspase-3 positive cells (green) was notably increased in the hippocampus of $d b / d b$ mice when compared with $\mathrm{db} / \mathrm{m}$ mice (Fig. 4e-g). Importantly, DL-NBP treatment significantly reversed these phenomena (Fig. $4 \mathrm{e}-\mathrm{g}$ ). These results suggest that DL-NBP treatment reduces neuronal apoptosis in the hippocampus of $d b / d b$ mice.

DL-NBP attenuates diabetes-induced oxidative stress in the hippocampus

We also investigated whether oxidative stress was present during DACD and whether DL-NBP treatment could prevent it. We found that NOX2 (Fig. 5a, b) and NOX4 (NADPH oxidase family members) 

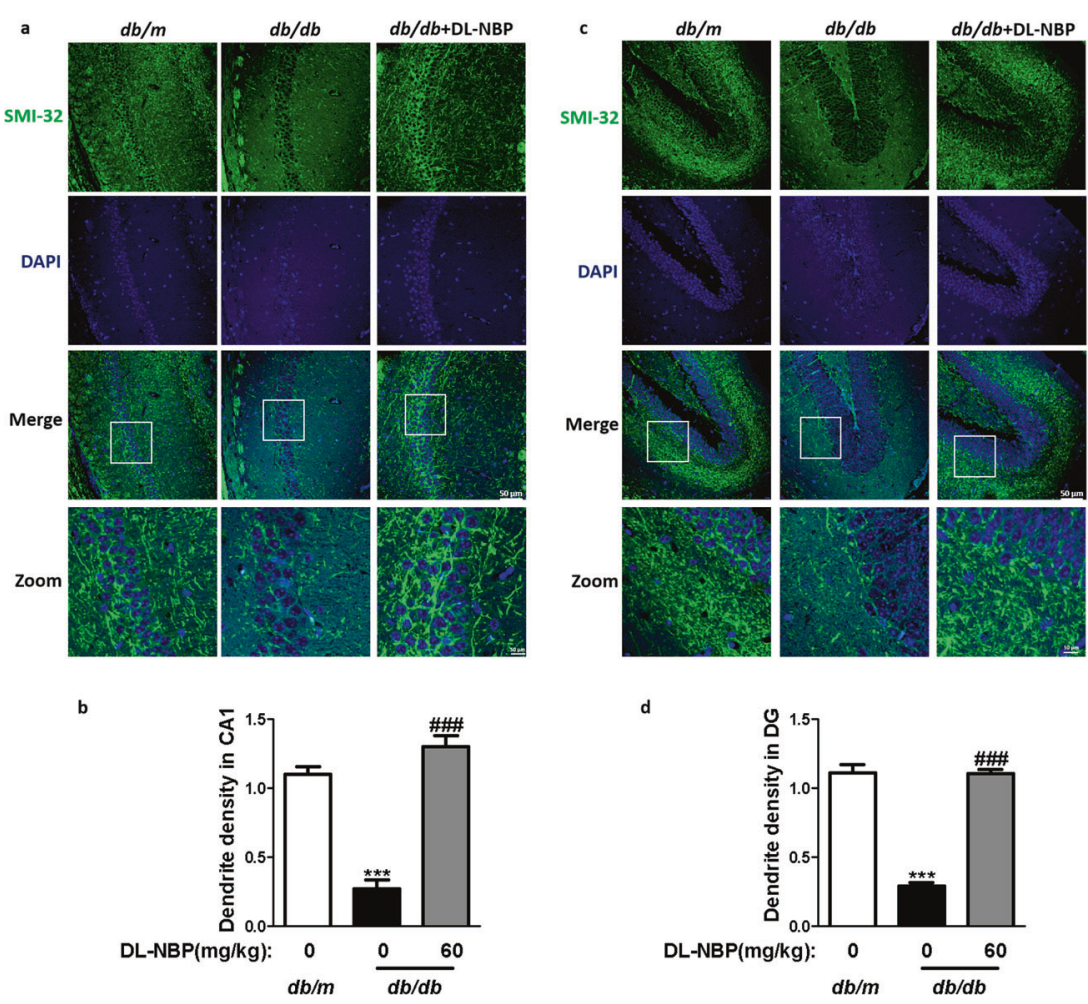

Fig. 3 DL-NBP improves the density and length of synapse in the hippocampus of $\boldsymbol{d b} / \boldsymbol{d} \boldsymbol{b}$ mice. a Immunofluorescence staining of SMI-32 in the CA1 region of the hippocampus. Scale bar $=50 \mu \mathrm{m}, n=3$. b Quantitative analysis of SMI-32 in the CA1 region of the hippocampus. c Immunofluorescence staining of SMI-32 in the DG region of the hippocampus. Scale bar $=50 \mu \mathrm{m}, n=3$. d Quantitative analysis of SMI-32 in the DG regions of the hippocampus. ${ }^{* *} P<0.001$ indicates $d b / m$ group vs. $d b / d b$ group; ${ }^{\# \#} P<0.001$ indicates DL-NBP $+d b / d b$ group vs. $d b / d b$ group.
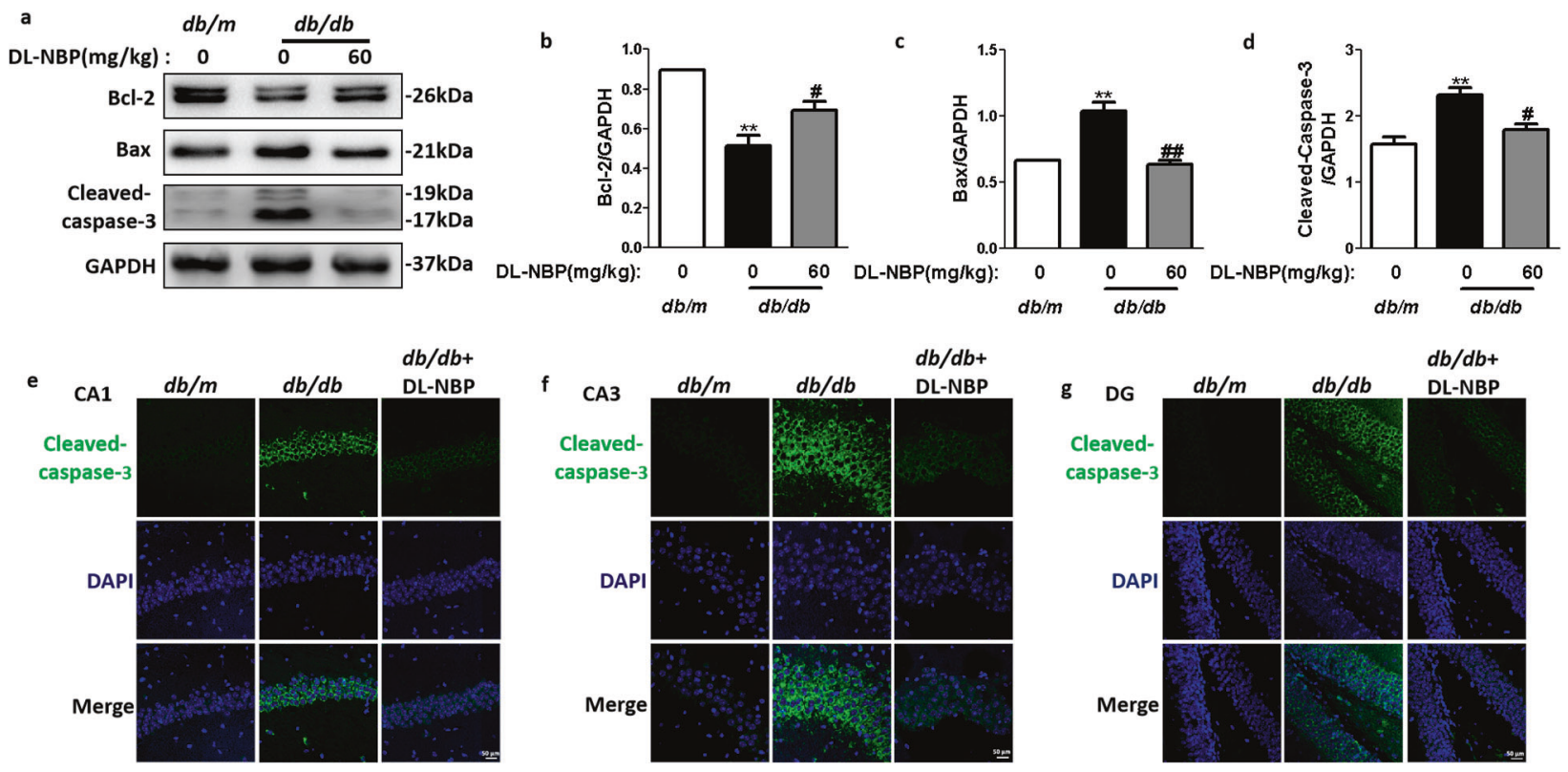

Fig. 4 DL-NBP reduces neuronal apoptosis in the hippocampus of $\boldsymbol{d} \boldsymbol{b} / \boldsymbol{d} \boldsymbol{b}$ mice. a Western blot quantification of Bcl-2, Bax and Cleavedcaspase-3 expression. b-d Quantitative analysis of Bcl-2, Bax and Cleaved-caspase-3 expression, $n=3$. e-g Immunofluorescence staining of Cleaved-caspase-3 in the CA1 (e), CA3 (f) and DG (g) regions of the hippocampus. Scale bar $=50 \mu \mathrm{m}, n=5$. ${ }^{* *} P<0.01$ indicates $d b / m$ group vs. $d b / d b$ group; ${ }^{\#} P<0.05,{ }^{\# \#} P<0.01$ indicates $d b / d b$ group vs. DL-NBP $+d b / d b$ group.

(Fig. 5a, c) protein expression levels were considerably enhanced in the hippocampus of $d b / d b$ mice group when compared with $d b / m$ mice, and that DL-NBP treatment substantially reduced those levels. We also assessed ROS levels by means of DHE staining assays. As shown in Fig. $5 \mathrm{~d}$, e, a robust DHE-positive signal was observed in the hippocampus of $d b / d b$ mice. DL-NBP treatment significantly reduced this signal. In addition, we evaluated the antioxidant properties of DL-NBP during DACD 

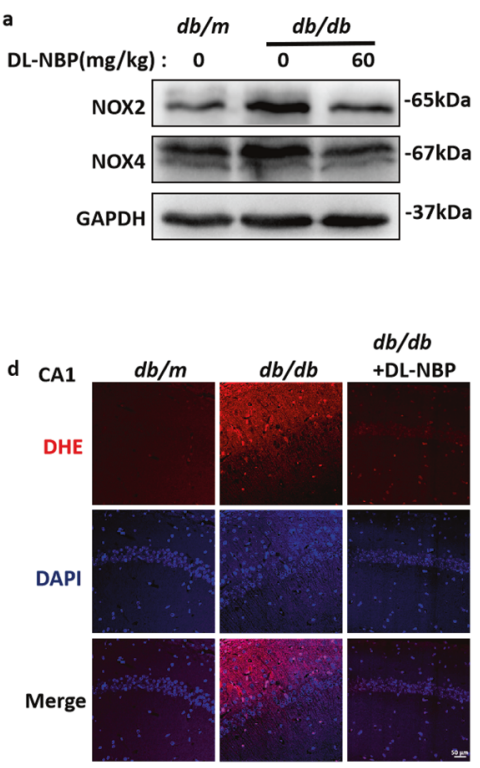
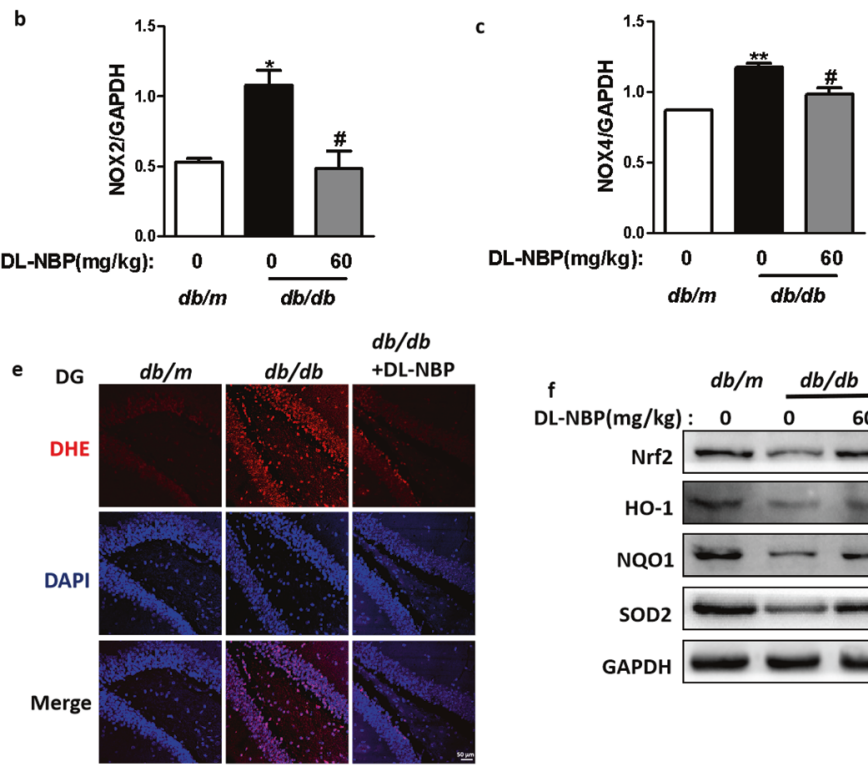

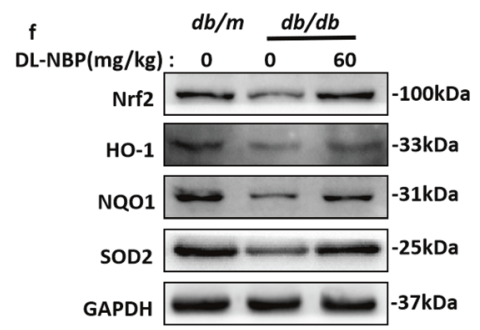

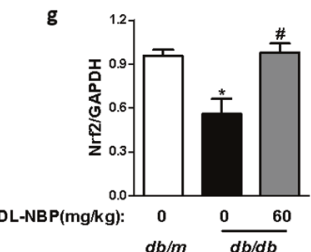

h
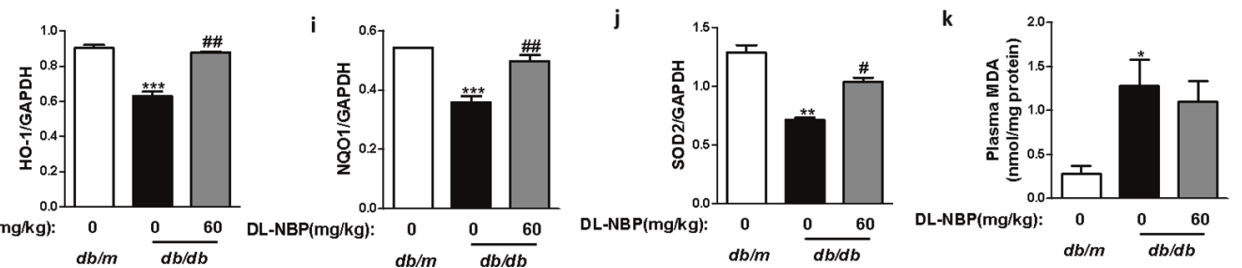

Fig. 5 DL-NBP attenuates diabetes-induced oxidative stress in the hippocampus. a Western blot quantification of NOX2 and NOX4 expression. $\mathbf{b}$ and $\mathbf{c}$ Quantitative analysis of NOX2 and NOX4 expression, $n=3$. $\mathbf{d}$ and e Representative images of DHE staining of the CA1 (d) and DG (e) regions of the hippocampus. Scale bar $=50 \mu \mathrm{m}, n=5$. $\mathbf{f}$ Western blot quantification of Nrf2, HO-1, NQO1 and SOD2 expression. g-j Quantitative analysis of Nrf2, HO-1, NQO1 and SOD2 expression, $n=3$. k Plasma MDA content in the different groups. $n=4$. ${ }^{*} P<0.05$, ${ }^{* *} P<0.01,{ }^{* *} P<0.001$ indicates $d b / m$ group vs. $d b / d b$ group; ${ }^{\#} P<0.05,{ }^{\# \#} P<0.01 d b / d b$ group vs. DL-NBP $+d b / d b$ group.

development. We found that the protein expression levels of Nrf2 (Fig. 5f, g) and related-antioxidants such as HO-1 (Fig. 5f, h), NQO1 (Fig. 5f, i) and SOD2 (Fig. 5f, j) were significantly reduced in the hippocampus of $d b / d b$ mice when compared with $\mathrm{db} / \mathrm{m}$ mice. DL-NBP treatment $(60 \mathrm{mg} / \mathrm{kg})$ significantly enhanced those protein expression levels. Moreover, DL-NBP treatment substantially reversed diabetes-induced elevations in the MDA content of the hippocampus (Fig. 5k). These results suggest that DL-NBP can inhibit oxidative stress in the hippocampus by inhibiting ROS production and activating the Nrf2 signaling pathway.

DL-NBP enhances BDNF levels in the hippocampus of $d b / d b$ mice by activating the Akt/CREB signaling pathway

Accumulating evidence indicates that BDNF, a neurotrophic factor, can promote immature neuron development and increase neuronal survival and synaptic plasticity [46]. CREB signaling regulates transcriptional expression of BDNF [21]. We investigated the effects of DL-NBP treatment on BDNF levels in the hippocampus. We found that BDNF (Fig. 6a, d), p-CREB (S133) (Fig. 6a, b) and p-Akt (Fig. 6a, c) protein expression levels were significantly reduced in the hippocampus of $d b / d b$ mice when compared with $\mathrm{db} / \mathrm{m}$ mice, and that DL-NBP treatment could reverse these reductions. Consistent with the Western blot results, DL-NBP treatment increased the intensity of BDNF positive signals in the CA1 and DG regions of the hippocampus in $d b / d b$ mice (Fig. 6e, f). These results demonstrate that DL-NBP enhances BDNF levels in the hippocampus of $d b / d b$ mice by activating Akt/CREB signaling.
DL-NBP reduces apoptosis of PC12 cells induced by high glucose Next, we investigated the effect of DL-NBP on PC12 cell apoptosis induced by high glucose (HG). We found that HG in the PC12 culture medium reduced the expression of the anti-apoptotic protein (BCl-2) (Fig. 7a, b) while enhancing expression of the proapoptotic protein (Bax) (Fig. 7a, c). Importantly, DL-NBP substantially reversed $\mathrm{HG}$-induced apoptosis of $\mathrm{PC} 12$ cells. Consistent with the Western blot results, immunofluorescence staining showed that Cleaved-caspase-3 level was increased in HG-exposed PC12 cells, but that DL-NBP significantly inhibited the Cleaved-caspase3 signal intensity (Fig. 7d). These findings indicate that DL-NBP can substantially inhibit apoptosis in HG-exposed PC12 cells.

DL-NBP alleviates high glucose-induced oxidative stress in PC12 cells through Nrf2 signaling pathways

We observed that DCFH-DA fluorescence intensity in the HG + DLNBP group was significantly lower than that in the HG group (Fig. 8a). Moreover, DL-NBP treatment significantly decreased NOX2 (Fig. 8b, c) and NOX4 (Fig. 8b, d) protein expression levels in HGincubated PC12 cells. These results further confirm the antioxidant properties of DL-NBP in HG-incubated PC12 cells. In addition, immunofluorescence staining showed that DL-NBP considerably enhanced the Nrf2 signal intensity in HG-incubated PC12 cells (Fig. 8e). More importantly, Nrf2 (Fig. 8f, g) protein expression levels and nuclear Nrf2 (Fig. 8f, h) levels in PC12 cells exposed to HG were reduced, but DL-NBP treatment increased these expression levels. Downstream proteins, such as HO-1 (Fig. 8i, j), NQO1 (Fig. 8i, k) and SOD2 (Fig. 8i, I), were downregulated in HG-incubated PC12 cells when compared with those in CON group, but DL-NBP treatment 


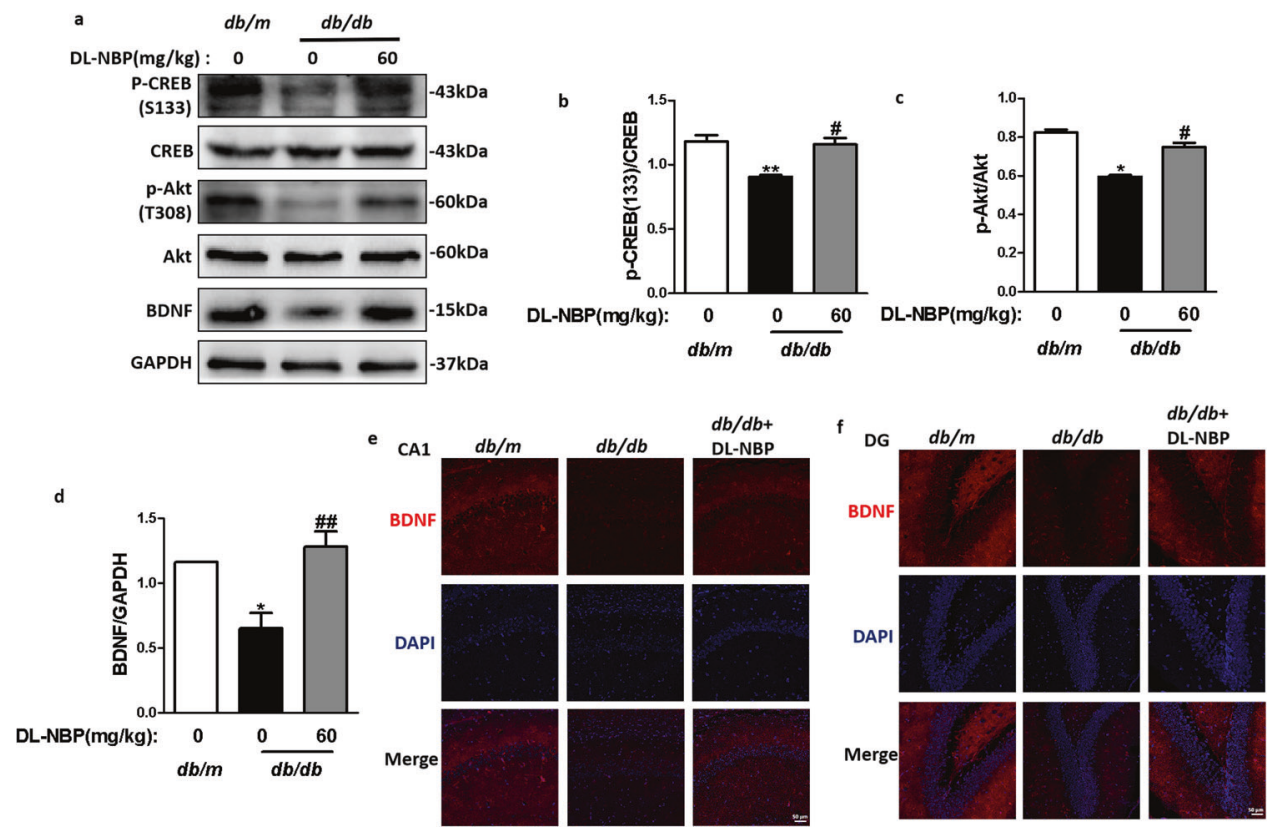

Fig. 6 DL-NBP enhances BDNF levels in the hippocampus of $d b / d b$ mice by activating the Akt/CREB signaling pathway. a Western blot quantification of p-CREB (S133), CREB, p-Akt, Akt and BDNF expression. b-d Quantitative analysis of p-CREB (S133), CREB, p-Akt, Akt and BDNF expression, $n=3$. e and $\mathbf{f}$ Immunofluorescence staining of BDNF in the CA1 (e) and DG (f) regions of the hippocampus. Scale bar $=$ $50 \mu \mathrm{m}, n=4$. ${ }^{*} P<0.05$, ${ }^{* *} P<0.01$ indicates $d b / m$ group vs. $d b / d b$ group; ${ }^{\#} P<0.05,{ }^{\# \#} P<0.01$ indicates $d b / d b$ group vs. DL-NBP $+d b / d b$ group.
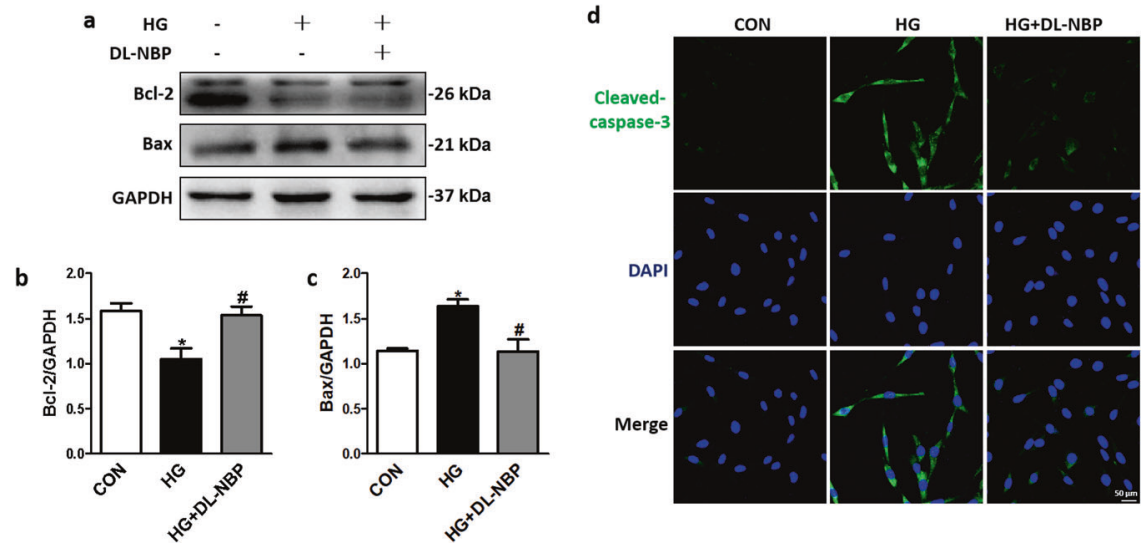

Fig. 7 DL-NBP reduces high glucose-induced apoptosis of PC12 cells. a Western blot analysis of Bcl-2 and Bax expression. b and c Quantitative analysis of Bcl-2 and Bax expression, $n=3$. d Immunofluorescence staining of Cleaved-caspase-3 in PC12 cells. Scale bar $=$ $50 \mu \mathrm{m}, n=5$. ${ }^{*} P<0.05$ indicates HG group vs. CON group; ${ }^{\#} P<0.05$ indicates HG+DL-NBP vs. HG group.

enhanced the expression levels of these proteins. These findings indicate that DL-NBP can alleviate oxidative stress in HG-incubated PC12 cells.

To investigate whether DL-NBP attenuated oxidative damage in HG-incubated PC12 cells by acting through Nrf2 signaling pathway, ML385, a Nrf2 inhibitor, was added to the culture medium. As shown in Fig. 9a, 9b, Nrf2 protein expression levels in the HG + DL-NBP group were significantly reduced after adding ML385 at 5, 10 and $20 \mu \mathrm{M}$. We finally selected $5 \mu \mathrm{M}$ of ML385 to perform the subsequent experiments. We found that DCFH-DA fluorescence intensity in the HG + DL-NBP + ML385 group was significantly higher than in the HG + DL-NBP group (Fig. 9c). We also found that Nrf2 (Fig. 9d, e), HO-1 (Fig. 9d, f), NQO1 (Fig. 9d, g) and SOD2 (Fig. 9d, h) expression levels were reduced in the HG + DL-NBP + ML385 group when compared with the HG + DL-NBP group. Finally, immunofluorescence staining showed that ML385 significantly increased the Cleaved-caspase-3 signal intensity in HG-exposed
PC12 cells treated with DL-NBP (Fig. 9i). Consistent with the immunofluorescence results, TUNEL-positive cells were significantly more abundant in the HG + DL-NBP + ML385 group than in the $\mathrm{HG}+\mathrm{DL}-\mathrm{NBP}$ group (Fig. 9j). These findings suggest that DL-NBP alleviates HG-induced oxidative stress in PC12 cells by acting the Nrf2 signaling pathway.

DL-NBP suppresses high glucose-induced oxidative stress and reverses downregulation of BDNF level in PC12 cells by acting $\mathrm{PI} 3 \mathrm{~K} /$ Akt signaling pathway

To investigate the potential role of PI3K/Akt signaling pathways in the effects of DL-NBP in HG-exposed PC12 cells, a PI3K inhibitor (LY294002) was added to the culture medium. In the Fig. 10a, we assessed the Nrf2 and its downstream proteins by Western blot, we found that the LY294002-treated group significantly reversed the DLNBP-induced upregulation of Nrf2 (Fig. 10a, b), HO-1(Fig. 10a, c), NQO1 (Fig. 10a, d) and SOD2 (Fig. 10a, e) in HG-exposed PC12 cells. 

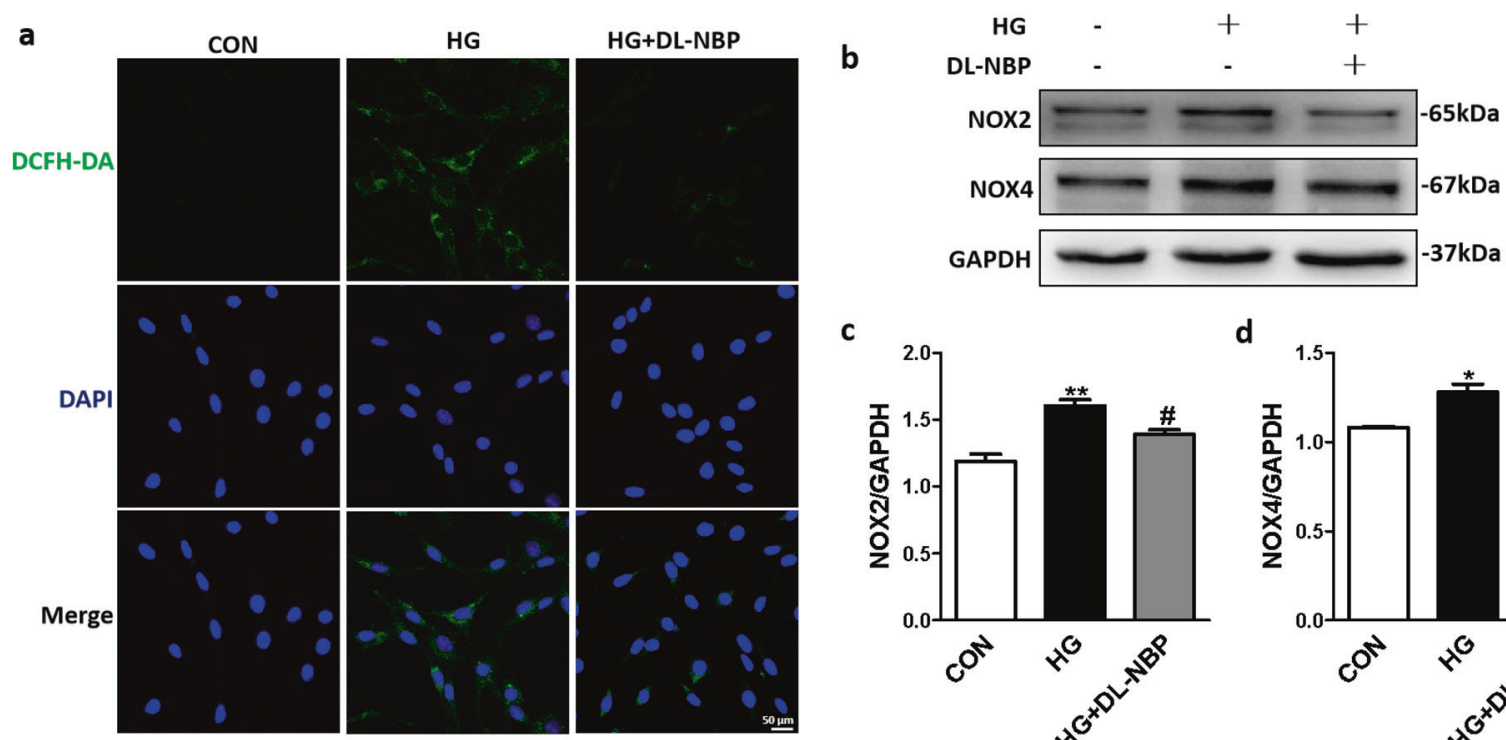

e
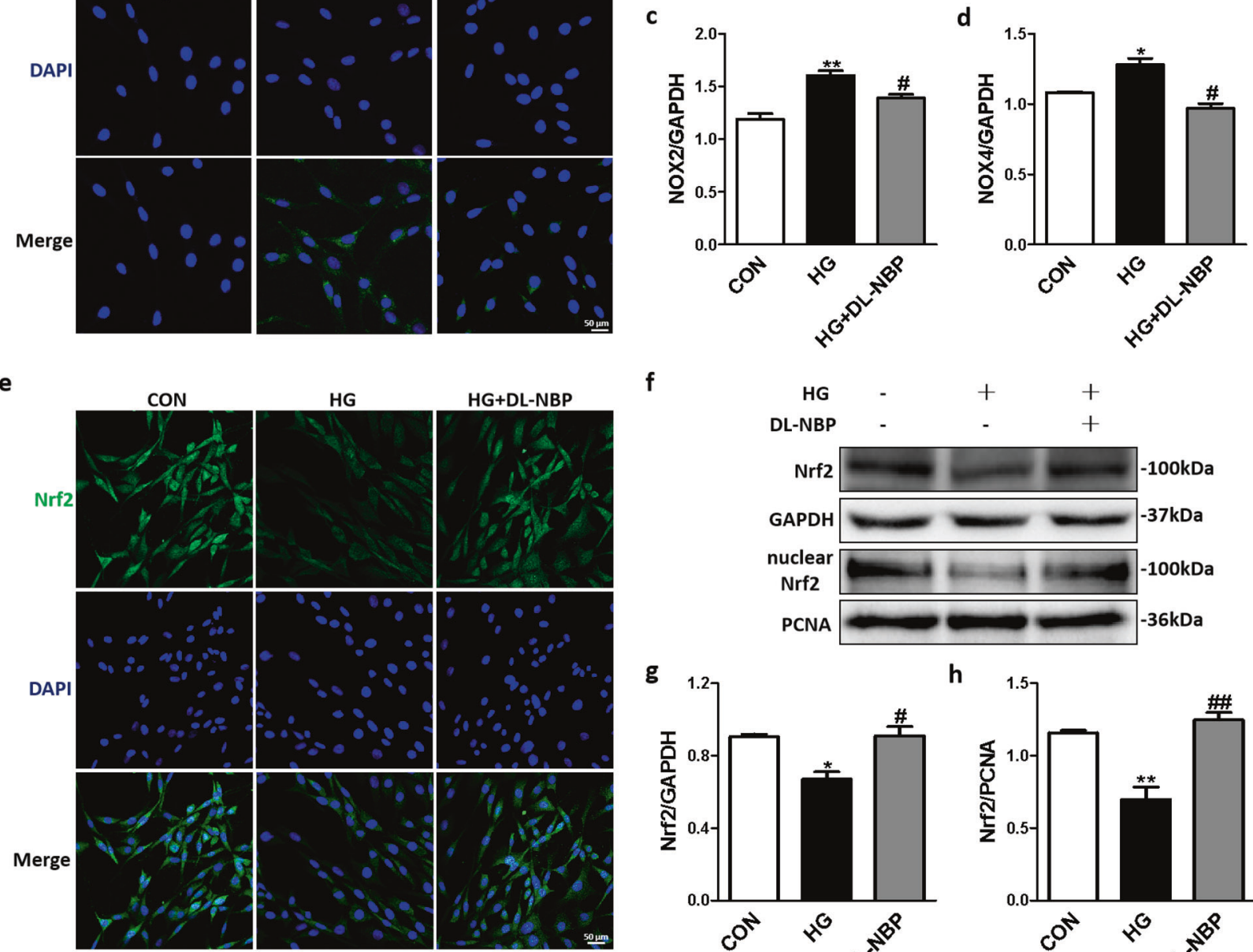

f

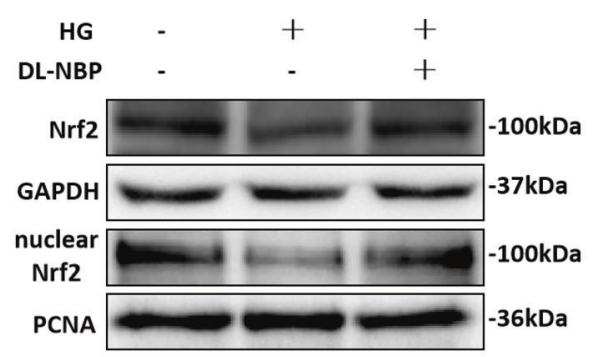

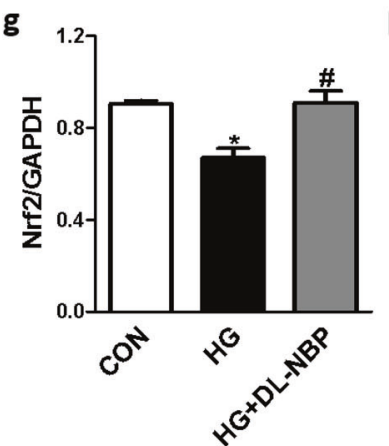

k

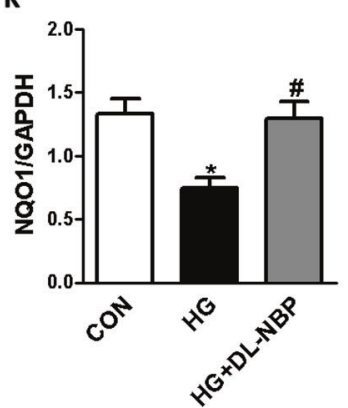

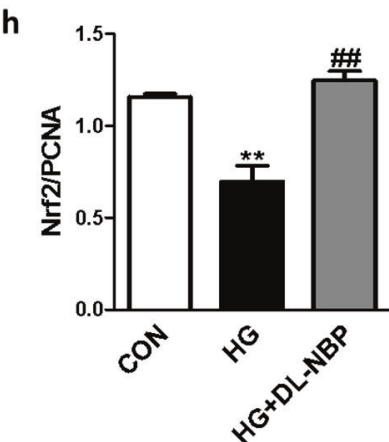

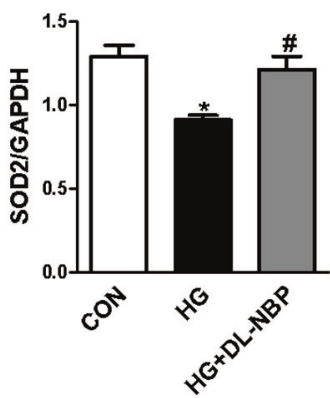

Fig. 8 DL-NBP reduces apoptosis of PC12 cells induced by high glucose levels. a Detection of ROS by immunofluorescence with dichlorodihydrofluorescein diacetate (DCFH-DA) in PC12 cells. Scale bar $=50 \mu \mathrm{m}, n=5$. b Western blot analysis of NOX2 and NOX4 expression. c and $\mathbf{d}$ Quantitative analysis of NOX2 and NOX4 expression, $n=3$. e Immunofluorescence staining of Nrf2 in PC12 cells. Scale bar $=50 \mu \mathrm{m}$, $n=5$. $\mathbf{f}$ Western blot analysis of Nrf2 and nuclear Nrf2 expression. $\mathbf{g}$ and $\mathbf{h}$ Quantitative analysis of Nrf2, and nuclear Nrf2 expression, $n=3$. i Western blot analysis of HO-1, NQO1 and SOD2 expression. j-I Quantitative analysis of HO-1, NQO1 and SOD2 expression, $n=3$. * $P<0.05$, ${ }^{* *} P<0.01$ indicates HG group vs. CON group; ${ }^{\#} P<0.05,{ }^{\# \#} P<0.01$ indicates HG+DL-NBP vs. HG group. 

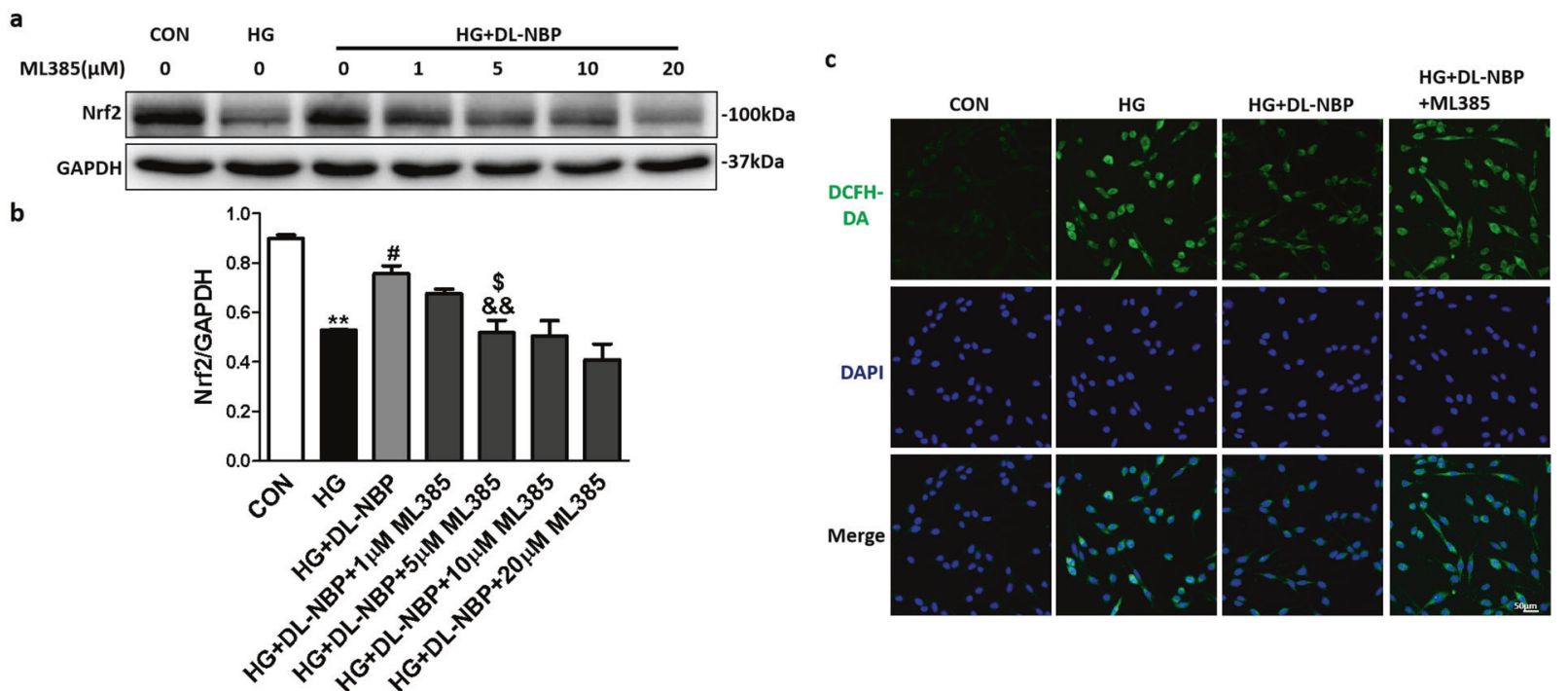
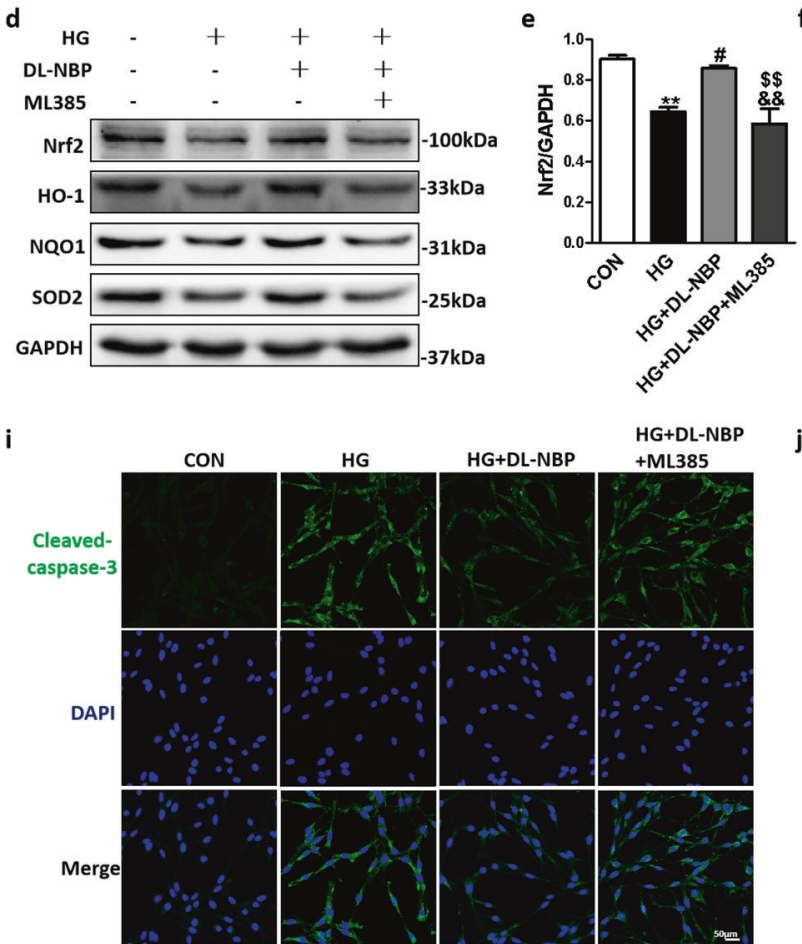
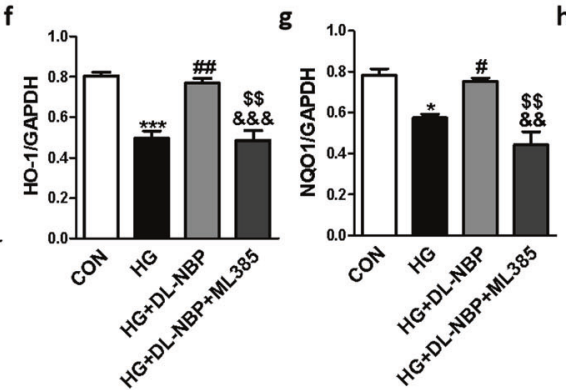

h

j

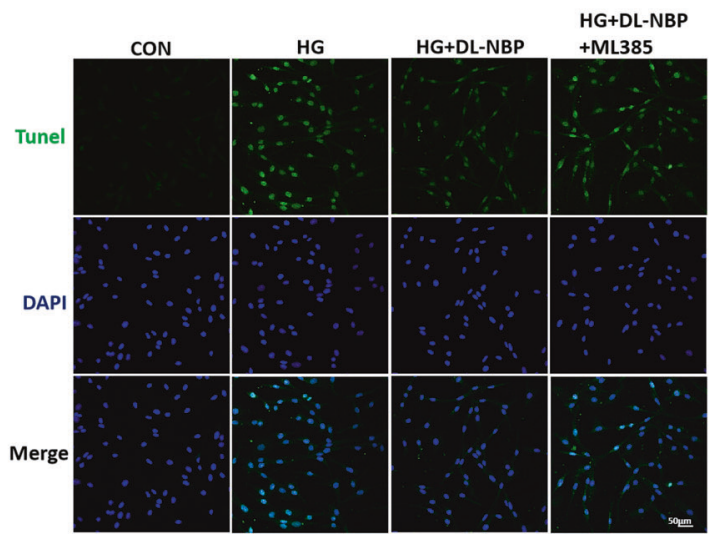

Fig. 9 DL-NBP alleviates high glucose-induced oxidative stress in PC12 cells through Nrf2 signaling pathway. a Western blot analysis of Nrf2 expression. b Quantitative analysis of Nrf2 expression, $n=3$. c Detection of ROS in PC12 cells by immunofluorescence with dichlorodihydrofluorescein diacetate (DCFH-DA). Scale bar $=50 \mu \mathrm{m}, n=3$. d Western blot analysis of Nrf2, HO-1, NQO1 and SOD2 expression. e-h Quantitative analysis of Nrf2, HO-1, NQO1 and SOD2 expression, $n=3$. i Immunofluorescence staining of Cleaved-caspase-3 in PC12 cells. Scale bar $=50 \mu \mathrm{m}, n=3$. j TUNEL staining of PC12 cells. Scale bar $=50 \mu \mathrm{m}, n=3$. ${ }^{*} P<0.05, * * P<0.01, * * * P<0.001$ indicates HG group vs. CON group; ${ }^{\#} P<0.05,{ }^{\# \#} P<0.01$ indicates HG+DL-NBP vs. HG group; ${ }^{\&} P<0.01$, \&\&\& $P<0.001$ indicates CON group vs. HG + DL-NBP+ML385 group; ${ }^{\$} P<0.05,{ }^{\$ \$} P<0.01$ indicates HG+DL-NBP group vs. HG+DL-NBP+ML385 group.

Next, we tested the Akt and related downstream proteins, as shown in Fig. 10f, g, p-Akt protein expression levels were decreased in the HG group, but these levels were restored by DL-NBP-treatment. In addition, LY294002 significantly reversed the DL-NBP-induced upregulation of p-Akt in HG-exposed PC12 cells (Fig. 10f, g). The pCREB (S133)/CREB ratio (Fig. 10f, h) and BDNF (Fig. 10f, i) expression levels showed the same trend. In addition, immunofluorescence staining showed that DL-NBP significantly reduced Cleaved-caspase-3 signal intensity in HG-incubated PC12 cells, but LY294002 partially reversed this effect (Fig. 10j). Consistent with the immunofluorescence results, LY294002 significantly inhibited the effects of DL-NBP on Bcl-2
(Fig. 10k, I) and Bax (Fig. 10k, m) protein expression levels in HGincubated PC12 cells. These findings suggest that PI3K/Akt pathways are essential for DL-NBP-induced signaling and for enhancing BDNF expression levels in HG-incubated PC12 cells.

\section{DISCUSSION}

Diabetes causes structural and functional changes in the central nervous system, resulting in cognitive decline $[47,48]$. The level of hyperglycemia and duration of diabetes have been linked to the degree of cognitive decline. It is essential to elucidate the exact 

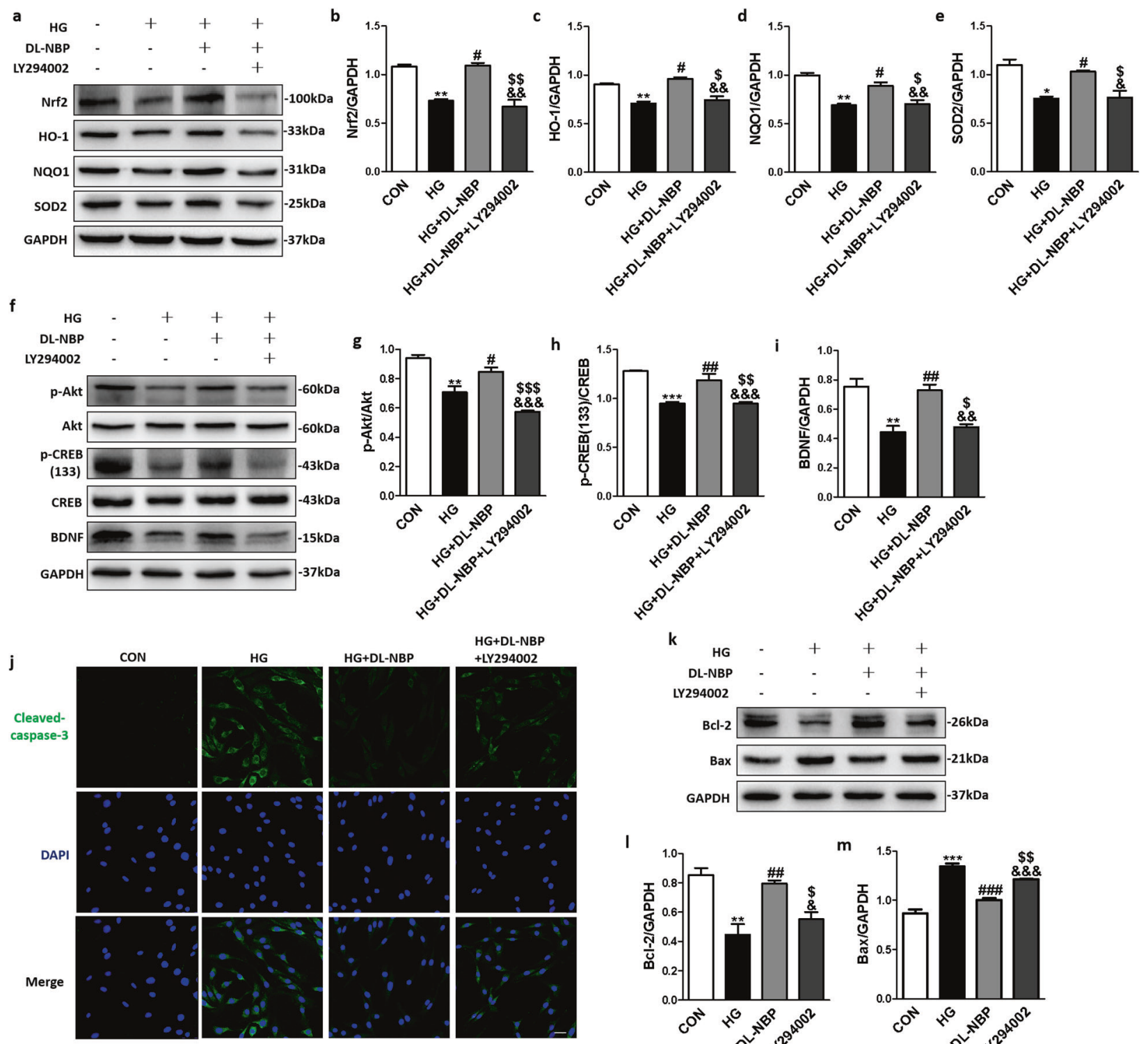

HG+DL-NBP
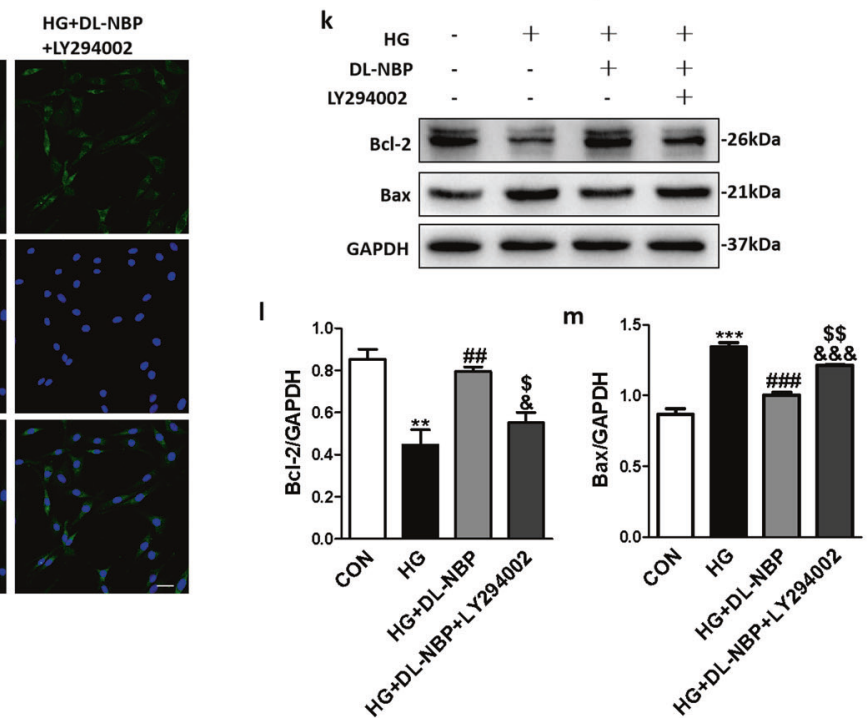

Fig. 10 DL-NBP suppresses high glucose-induced oxidative stress and reverses downregulation of BDNF level in PC12 cells by acting PI3K/Akt signaling pathway. a Western blot analysis of Nrf2, HO-1, NOO1 and SOD2 expression. b-e Quantitative analysis of Nrf2, HO-1, NQO1 and SOD2 expression, $n=3$. f Western blot analysis of p-Akt, Akt, p-CREB, CREB and BDNF expression. g-i Quantitative analysis of p-Akt, Akt, p-CREB, CREB and BDNF expression, $n=3$. j Immunofluorescence staining of Cleaved-caspase-3 in PC12 cells. Scale bar $=50 \mu \mathrm{m}, n=5$. $\mathbf{k}$ Western blot analysis of $\mathrm{Bcl}-2$ and Bax expression. $\mathbf{I}$ and $\mathbf{m}$ Quantitative analysis of $\mathrm{Bcl}-2$ and Bax expression, $n=3$. ${ }^{*} P<0.05,{ }^{* *} P<0.01$, ${ }^{* * *} P<0.001$ indicates HG group vs. CON group; ${ }^{\#} P<0.05$, ${ }^{\# \#} P<0.01,{ }^{\# \# \#} P<0.001$ indicates $\mathrm{HG}+\mathrm{DL}-\mathrm{NBP}$ vs. HG group; \& $P<0.05$, \&\& $P<0.01$, ${ }^{\& \& \&} P<0.001$ indicates CON group vs. HG+DL-NBP+LY294002 group; $P<0.05,{ }^{\$ \$} P<0.01, \$ \$ \$ P<0.001$ indicates HG+DL-NBP group vs. HG $+D L-$ NBP + LY294002 group.

molecular mechanisms underlying DACD development, and to identify reasonable and effective strategies for treating it. In the present study, we found that $d b / d b$ mice developed cognitive dysfunction, with impaired learning and memory. Oxidative stress and inhibition of the PI3K/Akt/CREB signaling pathway are the two main molecular mechanisms underlying DACD development.

DL-NBP is widely used for the treatment of ischemic stroke [49]. Multiple studies have shown that DL-NBP exerts significant neuroprotective effects in cerebral ischemia probably due to its ability to decrease brain edema, and thereby suppress oxidative stress and reduce neuronal death and inflammation [50]. It has also been reported that DL-NBP enhances cognitive function in
$A D$ and cerebral ischemia-reperfusion injury [51, 52]. Moreover, previous studies reported that DL-NBP significantly attenuated learning and memory deficits in diabetic mice [53]. Consistent with the prior study, our current study showed that DL-NBP reduced cognitive impairment in $d b / d b$ mice, as evidenced by shorter escape latencies and more numbers of platform crossings.

The hippocampus, an important functional area of the brain, is divided into three regions: $C A 1, C A 3$, and dentate gyrus (DG). The hippocampus is involved in the regulation of short-term memory, learning, executive ability and attention, especially the CA 1 region [54]. It has been shown that diabetes alters the structure and function of neurons and synapses in the CA1 region, thereby 
affecting synaptic plasticity and long-term potentiation (LTP) $[55,56]$. Thus, we focused on the hippocampus, and found that DL-NBP administration to $d b / d b$ mice ameliorated the structural alterations, loss of neurons and suppression of synaptic-related proteins seen in this brain structure in DACD, improving spatial learning and memory. This result is also consistent with previous studies reporting that treatment with DL-NBP significantly improved hippocampal tissue morphology in diabetic mice [53].

Oxidative stress is associated with several diabetes-associated complications $[57,58]$. Oxidative damage also plays a central role in vascular cognitive impairment in dementia patients and $A D$ models [59]. In addition, previous research showed that the neuroprotective effect of DL-NBP in an AD mouse model depended on upregulation of the antioxidant defenses [34]. However, previous studies have not described in detail the possible mechanisms by which DL-NBP improves cognitive impairment in diabetes. We speculated that oxidative stress was the key molecular mechanism underlying DACD, and that DL-NBP treatment could prevent it. In this study, we found that diabetes induced oxidative stress, with elevated levels of ROS, lipid peroxidation products, and NADPH oxidases. The antioxidant capacity was also disrupted by suppression of $\mathrm{Nrf} 2 / \mathrm{HO}-1 /$ NQO1 signaling pathways in the hippocampus of $d b / d b$ mice. Importantly, DL-NBP could reverse these alterations. We also used ML385, a Nrf2 inhibitor, to confirm the previous conclusions. These results indicate that DL-NBP can significantly impact the pathogenesis and progression of DACD by exerting antioxidative effects.

Previous studies have shown that Akt can regulate Nrf2 signals in PC12 cells and zebrafish to protect against 6-OHDA-induced neurotoxicity [60]. Other studies have also suggested that PI3K/Akt can regulate $\mathrm{Nrf2}$ to improve cognitive dysfunction and neurological deficits in $D$-galactose-induced aging mice [61]. Hence, we speculated that the neuroprotective effects of DL-NBP in $d b / d b$ mice were partly mediated by activation of PI3K/Akt/ Nrf2 signaling. In our current study, we used the PI3K inhibitor LY294002 and found that DL-NBP enhanced anti-oxidative capacities by regulating $\mathrm{PI} 3 \mathrm{~K} / \mathrm{Akt} / \mathrm{Nrf} 2$ signaling.

Neurogenesis dysfunction is considered to be an important causative event in cognitive impairment $[62,63]$. Neurotrophic factors play a crucial role in neurogenesis [64]. Therefore, stimulating neurotrophic factors have been regarded as a potential therapeutic strategy to delay or halt cognitive decline. BDNF is an important neurotrophic factor, which enhances synapse formation and cognitive function. The neuroprotective effect of BDNF is dependent on CREB-mediated transcription $[65,66]$. In our current study, we found that diabetes inhibited CREB activity and consequently BDNF expression in the hippocampus, and that DL-NBP reversed these alterations. The PI3K/Akt signaling pathway participates in neuronal differentiation, survival, glucose metabolism, and synaptic function. In addition, Akt can phosphorylate several survival regulatory molecules, including CREB. It has been reported that the PI3K/Akt signaling pathway is inhibited in AD [67, 68]. Moreover, DL-NBP administration promotes activation of the PI3K/Akt signaling pathway [69]. However, whether DL-NBP could reverse DACD by regulating this pathway was unknown. In our current study, we found that the $\mathrm{PI} 3 \mathrm{~K} / \mathrm{Akt} / \mathrm{CREB}$ signaling pathway mediated the neuroprotective effect of DL-NBP in DACD.

Although these results clearly illuminate some molecular mechanisms underlying the beneficial effects of DL-NBP in DACD development, the target of DL-NBP in the hippocampus is still unclear. Increased blood-brain barrier (BBB) permeability is a critical neurovascular complication of T2D that adversely affects central nervous system homeostasis and function [70]. The destruction of the BBB may be an important trigger of complex cascades of molecular events during DACD. Our own and other studies have reported that DL-NBP can restore BBB permeability

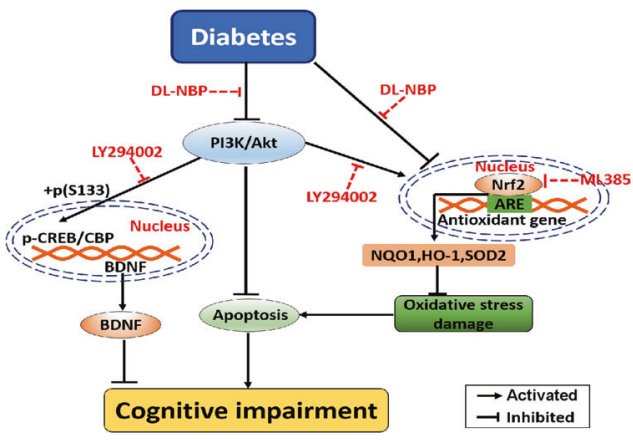

Fig. 11 Schematic diagram showing how DL-NBP ameliorates diabetes-induced cognitive dysfunction.

after traumatic brain injury and cerebral ischemia-reperfusion injury $[71,72]$. Hence, we hypothesize that the BBB may be a key target of DL-NBP in DACD. Several potential molecular mechanisms may be involved, such as anti-inflammatory and angiogenesis effects, and future studies are needed to clarify this point.

In summary, this study showed that DL-NBP exerts substantial neuroprotective effects and inhibits cognitive impairment in DACD by normalizing hippocampal morphology, improving synaptic plasticity, and reducing neuronal apoptosis. Multiple studies have demonstrated that inhibition of oxidative stress and activation of $\mathrm{PI}$ I3K/Akt/CREB signaling pathways are important mechanisms underlying the effects of DL-NBP in DACD (Fig. 11). Therefore, these findings suggest that DL-NBP may be a potential therapeutic agent for the treatment of DACD.

\section{ACKNOWLEDGEMENTS}

This study is supported by the research grants from the National Natural Science Foundation of China $(81801233,81870842,81801245,81802238)$ and Natural Science Foundation of Zhejiang Province (LQ18H090011 and LGD21H070001).

\section{AUTHOR CONTRIBUTIONS}

BNW, CBW and YQW did the research and wrote the paper. ZMC participated in data analysis and writing the paper. PPZ, YQL, JX, JYX, PFL, AAM, LBY and ZLZ researched data. JX and JW conceived the project, designed the experiments, and wrote the paper. All authors have approved the final version of the paper.

\section{ADDITIONAL INFORMATION}

The online version of this article (https://doi.org/10.1038/s41401-020-00583-3) contains supplementary material, which is available to authorized users.

Competing interests: The authors declare no competing interests.

\section{REFERENCES}

1. Enomoto M, Yoshii H, Mita T, Sanke H, Yokota A, Yamashiro K, et al. Relationship between dietary pattern and cognitive function in elderly patients with type 2 diabetes mellitus. J Int Med Res. 2015;43:506-17.

2. Cooray GK, Maurex L, Brismar T. Cognitive impairment correlates to low auditory event-related potential amplitudes in type 1 diabetes. Psychoneuroendocrinology. 2008;33:942-50.

3. Plastino M, Fava A, Pirritano D, Cotronei P, Sacco N, Sperli T, et al. Effects of insulinic therapy on cognitive impairment in patients with Alzheimer disease and diabetes mellitus type-2. J Neurol Sci. 2010;288:112-6.

4. Koekkoek PS, Ruis C, van den Donk M, Biessels GJ, Gorter KJ, Kappelle LJ, et al. Intensive multifactorial treatment and cognitive functioning in screen-detected type 2 diabetes-the addition-netherlands study: a cluster-randomized trial. J Neurol Sci. 2012;314:71-7.

5. Aly HF, Mantawy MM. Comparative effects of zinc, selenium and vitamin E or their combination on carbohydrate metabolizing enzymes and oxidative stress in streptozotocin induced-diabetic rats. Eur Rev Med Pharmacol Sci. 2012;16: 66-78. 
6. Reddy PH. Amyloid precursor protein-mediated free radicals and oxidative damage: implications for the development and progression of Alzheimer's disease. J Neurochem. 2006;96:1-13.

7. Fukui K, Onodera K, Shinkai T, Suzuki S, Urano S. Impairment of learning and memory in rats caused by oxidative stress and aging, and changes in antioxidative defense systems. Ann Ny Acad Sci. 2001;928:168-75.

8. Ansari MA, Scheff SW. Oxidative stress in the progression of Alzheimer disease in the frontal cortex. J Neuropathol Exp Neurol. 2010;69:155-67.

9. Peng Y, Hu Y, Xu S, Li P, Li J, Lu L, et al. L-3-n-butylphthalide reduces tau phosphorylation and improves cognitive deficits in AbetaPP/PS1-Alzheimer's transgenic mice. J Alzheimers Dis. 2012;29:379-91.

10. Baydas G, Donder E, Kiliboz M, Sonkaya E, Tuzcu M, Yasar A, et al. Neuroprotection by alpha-lipoic acid in streptozotocin-induced diabetes. Biochemistry (Mosc). 2004;69:1001-5.

11. Li W, Jain MR, Chen C, Yue X, Hebbar V, Zhou R, et al. Nrf2 possesses a redoxinsensitive nuclear export signal overlapping with the leucine zipper motif. J Biol Chem. 2005;280:28430-8.

12. Gong W, Li J, Zhu G, Wang Y, Zheng G, Kan Q. Chlorogenic acid relieved oxidative stress injury in retinal ganglion cells through IncRNA-TUG1/Nrf2. Cell Cycle. 2019;18:1549-59.

13. Singh N, Vijayanti S, Saha L, Bhatia A, Banerjee D, Chakrabarti A. Neuroprotective effect of Nrf2 activator dimethyl fumarate, on the hippocampal neurons in chemical kindling model in rat. Epilepsy Res. 2018;143:98-104.

14. Shi Y, Miao W, Teng J, Zhang L. Ginsenoside Rb1 protects the brain from damage induced by epileptic seizure via Nrf2/ARE signaling. Cell Physiol Biochem. 2018:45:212-25.

15. Zhang Y, Liu B, Chen X, Zhang N, Li G, Zhang LH, et al. Naringenin ameliorates behavioral dysfunction and neurological deficits in a $D$-galactose-induced aging mouse model through activation of PI3K/Akt/Nrf2 pathway. Rejuvenation Res. 2017;20:462-72.

16. Frebel K, Wiese S. Signalling molecules essential for neuronal survival and differentiation. Biochem Soc Trans. 2006;34:1287-90.

17. Ali T, Kim T, Rehman SU, Khan MS, Amin FU, Khan $M$, et al. Natural dietary supplementation of anthocyanins via PI3K/Akt/Nrf2/HO-1 pathways mitigate oxidative stress, neurodegeneration, and memory impairment in a mouse model of Alzheimer's disease. Mol Neurobiol. 2018;55:6076-93.

18. Nakaso K, Yano H, Fukuhara Y, Takeshima T, Wada-Isoe K, Nakashima K. PI3K is a key molecule in the Nrf2-mediated regulation of antioxidative proteins by hemin in human neuroblastoma cells. FEBS Lett. 2003;546:181-4.

19. Wang L, Chen Y, Sternberg P, Cai J. Essential roles of the PI3 kinase/Akt pathway in regulating Nrf2-dependent antioxidant functions in the RPE. Invest Ophthalmol Vis Sci. 2008:49:1671-8.

20. Wen Z, Hou W, Wu W, Zhao Y, Dong X, Bai X, et al. 6'-O-Galloylpaeoniflorin attenuates cerebral ischemia reperfusion-induced neuroinflammation and oxidative stress via PI3K/Akt/Nrf2 activation. Oxid Med Cell Longev. 2018;2018: 8678267

21. Lopez de Armentia M, Jancic D, Olivares R, Alarcon JM, Kandel ER, Barco A. CAMP response element-binding protein-mediated gene expression increases the intrinsic excitability of CA1 pyramidal neurons. J Neurosci. 2007;27:13909-18.

22. AM L, NY I, MM P. Potentiation of developing neuromuscular synapses by the neurotrophins NT-3 and BDNF. Nature. 1993;363:350-3.

23. Figurov A, Pozzo-Miller LD, Olafsson P, Wang T, Lu B. Regulation of synaptic responses to high-frequency stimulation and LTP by neurotrophins in the hippocampus. Nature. 1996;381:706-9.

24. Phillips HS, Hains JM, Armanini M, Laramee GR, Johnson SA, Winslow JW. BDNF mRNA is decreased in the hippocampus of individuals with Alzheimer's disease. Neuron. 1991;7:695-702.

25. Li G, Peskind ER, Millard SP, Chi P, Sokal I, Yu CE, et al. Cerebrospinal fluid concentration of brain-derived neurotrophic factor and cognitive function in non-demented subjects. PLoS One. 2009:4:e5424.

26. Kariharan T, Nanayakkara G, Parameshwaran K, Bagasrawala I, Ahuja M, AbdelRahman E, et al. Central activation of PPAR-gamma ameliorates diabetes induced cognitive dysfunction and improves BDNF expression. Neurobiol Aging. 2015;36: 1451-61.

27. Chen J, Liang L, Zhan LB, Zhou Y, Zheng LP, Sun XX, et al. ZiBuPiYin recipe protects $d b / d b$ mice from diabetes-associated cognitive decline through improving multiple pathological changes. PLoS One. 2014;9:e91680.

28. Carvalho C, Machado N, Mota PC, Correia SC, Cardoso S, Santos RX, et al. Type 2 diabetic and Alzheimer's disease mice present similar behavioral, cognitive, and vascular anomalies. J Alzheimers Dis. 2013;35:623-35.

29. Peng B, Cui LY. Treatment for acute ischemic stroke: new evidence from China. Chin Med J (Engl). 2013;126:3403-4.

30. Hu JY, Wen QP, Wu Y, Li BZ, Gao P. The effect of butylphthalide on the brain edema, blood-brain barrier of rats after focal cerebral infarction and the expression of Rho A. Cell Biochem Biophys. 2014:69:363-8.
31. Jia JP, Wei CB, Liang JH, Zhou AH, Zuo XM, Song HG, et al. The effects of DL-3-nbutylphthalide in patients with vascular cognitive impairment without dementia caused by subcortical ischemic small vessel disease: a multicentre, randomized, double-blind, placebo-controlled trial. Alzheimers Dement. 2016;12: 89-99.

32. Xiong NA, Huang JS, Chen CN, Zhao Y, Zhang ZW, Jia M, et al. DL-3-n-butylphthalide, a natural antioxidant, protects dopamine neurons in rotenone models for Parkinson's disease. Neurobiol Aging. 2012;33:1777-91.

33. Yang LC, Li J, Xu SF, Cai J, Lei H, Liu DM, et al. L-3-n-butylphthalide promotes neurogenesis and neuroplasticity in cerebral ischemic rats. CNS Neurosci Ther 2015:21:733-41.

34. Wang CY, Wang ZY, Xie JW, Wang T, Wang X, Xu Y, et al. DL-3-n-butylphthalideinduced upregulation of antioxidant defense is involved in the enhancement of cross talk between CREB and Nrf2 in an Alzheimer's disease mouse model. Neurobiol Aging. 2016;38:32-46.

35. Abdoulaye IA, Guo YJ. A review of recent advances in neuroprotective potential of 3-n-butylphthalide and its derivatives. Biomed Res Int. 2016;2016: 5012341.

36. Peng Y, Xu SF, Chen GQ, Wang L, Feng YP, Wang XL. L-3-n-butylphthalide improves cognitive impairment induced by chronic cerebral hypoperfusion in rats. J Pharmacol Exp Ther. 2007;321:902-10.

37. Nicholas A, Munhoz CD, Ferguson D, Campbell L, Sapolsky R. Enhancing cognition after stress with gene therapy. J Neurosci. 2006;26:11637-43.

38. Xu Y, Pan J, Sun J, Ding L, Ruan L, Reed M, et al. Inhibition of phosphodiesterase 2 reverses impaired cognition and neuronal remodeling caused by chronic stress. Neurobiol Aging. 2015;36:955-70.

39. Chien T, Weng YT, Chang SY, Lai HL, Chiu FL, Kuo HC, et al. GSK3beta negatively regulates TRAX, a scaffold protein implicated in mental disorders, for NHEJmediated DNA repair in neurons. Mol Psychiatry. 2018;23:2375-90.

40. Meng L, Chen R, Jiang A, Wang L, Wang P, Li CZ, et al. Short multiwall carbon nanotubes promote neuronal differentiation of $\mathrm{PC} 12$ cells via up-regulation of the neurotrophin signaling pathway. Small. 2013;9:1786-98.

41. Danielyan L, Schafer R, Schulz A, Ladewig T, Lourhmati A, Buadze M, et al. Survival, neuron-like differentiation and functionality of mesenchymal stem cells in neurotoxic environment: the critical role of erythropoietin. Cell Death Differ 2009;16:1599-614.

42. Li M, Yu HB, Pan HY, Zhou XQ, Ruan QF, Kong DL, et al. Nrf2 suppression delays diabetic wound healing through sustained oxidative stress and inflammation. Front Pharmacol. 2019;10:1099.

43. Wang ZG, Zhang HY, Xu XL, Shi HX, Yu XC, Wang XJ, et al. bFGF inhibits ER stress induced by ischemic oxidative injury via activation of the PI3K/Akt and ERK1/2 pathways. Toxicol Lett. 2012;212:137-46.

44. Li L, Li T, Zhang Y, Pan Z, Wu B, Huang X, et al. Peroxisome proliferator-activated receptorbeta/delta activation is essential for modulating p-Foxo1/Foxo1 status in functional insulin-positive cell differentiation. Cell Death Dis. 2015;6:e1715.

45. Hu X, Shi Q, Zhou X, He W, Yi H, Yin X, et al. Transgenic mice overexpressing reticulon 3 develop neuritic abnormalities. EMBO J. 2007;26:2755-67.

46. Sairanen M, O'Leary OF, Knuuttila JE, Castren E. Chronic antidepressant treatment selectively increases expression of plasticity-related proteins in the hippocampus and medial prefrontal cortex of the rat. Neuroscience. 2007;144:368-74.

47. Li ZG, Zhang W, Sima AA. C-peptide enhances insulin-mediated cell growth and protection against high glucose-induced apoptosis in SH-SY5Y cells. Diabetes Metab Res Rev. 2003;19:375-85.

48. Sale P, De Pandis MF, Vimercati SL, Sova I, Foti C, Tenore N, et al. The relation between Parkinson's disease and ageing comparison of the gait patterns of young Parkinson's disease subjects with healthy elderly subjects. Eur J Phys Rehab Med. 2013:49:161-7.

49. Sun YF, Cheng X, Wang HB, Mu XP, Liang YF, Luo YJ, et al. DL-3-n-butylphthalide promotes neuroplasticity and motor recovery in stroke rats. Behav Brain Res. 2017;329:67-74.

50. Li JM, Li Y, Ogle M, Zhou X, Song MK, Yu SP, et al. DL-3-n-Butylphthalide prevents neuronal cell death after focal cerebral ischemia in mice via the JNK pathway. Brain Res. 2010;1359:216-26.

51. Ye XW, Rong ZY, Li YF, Wang XT, Cheng BY, Cheng YY, et al. Protective role of L-3n-butylphthalide in cognitive function and dysthymic disorders in mouse with chronic epilepsy. Front Pharmacol. 2018;9:734.

52. Xu J, Huai YP, Meng N, Dong YH, Liu ZJ, Qi QQ, et al. L-3-n-butylphthalide activates Akt/mTOR signaling, inhibits neuronal apoptosis and autophagy and improves cognitive impairment in mice with repeated cerebral ischemiareperfusion injury. Neurochem Res. 2017;42:2968-81.

53. Gao M, Ji S, Li J, Zhang S. DL-3-n-butylphthalide (NBP) ameliorates cognitive deficits and CaMKII-mediated long-term potentiation impairment in the hippocampus of diabetic $d b / d b$ mice. Neurol Res. 2019;41:1024-33.

54. Di Mario U, Morano S, Valle E, Pozzessere G. Electrophysiological alterations of the central nervous system in diabetes mellitus. Diabetes Metab Rev. 1995;11:259-77. 
55. Xiang Q, Zhang J, Li CY, Wang Y, Zeng MJ, Cai ZX, et al. Insulin resistance-induced hyperglycemia decreased the activation of Akt/CREB in hippocampus neurons: Molecular evidence for mechanism of diabetes-induced cognitive dysfunction. Neuropeptides. 2015;54:9-15.

56. Bliss TV, Collingridge GL. A synaptic model of memory: long-term potentiation in the hippocampus. Nature. 1993;361:31-9.

57. Dagon Y, Avraham Y, Link G, Zolotarev O, Mechoulam R, Berry EM. The synthetic cannabinoid HU-210 attenuates neural damage in diabetic mice and hyperglycemic pheochromocytoma PC12 cells. Neurobiol Dis. 2007;27:174-81.

58. Song $Y$, Ding W, Bei $Y$, Xiao $Y$, Tong HD, Wang LB, et al. Insulin is a potential antioxidant for diabetes-associated cognitive decline via regulating Nrf2 dependent antioxidant enzymes. Biomed Pharmacother. 2018;104:474-84.

59. Qi QQ, Xu J, Lv PY, Dong YH, Liu ZJ, Hu M, et al. DL-3-n-butylphthalide alleviates vascular cognitive impairment induced by chronic cerebral hypoperfusion by activating the Akt/Nrf2 signaling pathway in the hippocampus of rats. Neurosci Lett. 2018;672:59-64.

60. Zhang C, Li C, Chen S, Li Z, Jia X, Wang K, et al. Berberine protects against 6OHDA-induced neurotoxicity in PC12 cells and zebrafish through hormetic mechanisms involving $\mathrm{PI} 3 \mathrm{~K} / \mathrm{AKT} / \mathrm{BCl}-2$ and Nrf2/HO-1 pathways. Redox Biol. 2017;11:1-11.

61. Zhao M, Tang X, Gong D, Xia P, Wang F, Xu S. Bungeanum improves cognitive dysfunction and neurological deficits in $D$-galactose-induced aging mice via activating PI3K/Akt/Nrf2 signaling pathway. Front Pharmacol. 2020;11:71.

62. $\mathrm{Mu} \mathrm{YL,} \mathrm{Gage} \mathrm{FH.} \mathrm{Adult} \mathrm{hippocampal} \mathrm{neurogenesis} \mathrm{and} \mathrm{its} \mathrm{role} \mathrm{in} \mathrm{Alzheimer's}$ disease. Mol Neurodegener. 2011;6:85. https://doi.org/10.1186/1750-1326-6-85.

63. Tatebayashi Y, Lee MH, Li L, lqbal K, Grundke-lqbal I. The dentate gyrus neurogenesis: a therapeutic target for Alzheimer's disease. Acta Neuropathol. 2003:105:225-32.
64. Agrimi J, Spalletti C, Baroni C, Keceli G, Zhu GS, Caragnano A, et al. Obese mice exposed to psychosocial stress display cardiac and hippocampal dysfunction associated with local brain-derived neurotrophic factor depletion. Ebiomedicine. 2019;47:384-401.

65. Belgacem YH, Borodinsky LN. CREB at the crossroads of activity-dependent regulation of nervous system development and function. Adv Exp Med Biol. 2017;1015:19-39.

66. Gite S, Ross RP, Kirke D, Guiheneuf F, Aussant J, Stengel DB, et al. Nutraceuticals to promote neuronal plasticity in response to corticosterone-induced stress in human neuroblastoma cells. Nutr Neurosci. 2019;22:551-68.

67. de la Monte SM, Wands JR. Alzheimer's disease is type 3 diabetes-evidence reviewed. J Diabetes Sci Technol. 2008;2:1101-13.

68. Lee HK, Kumar P, Fu Q, Rosen KM, Querfurth HW. The insulin/Akt signaling pathway is targeted by intracellular beta-amyloid. Mol Biol Cell. 2009;20:1533-44.

69. Sun B, Feng MJ, Tian XY, Lu XW, Zhang YY, Ke XJ, et al. DL-3-n-butylphthalide protects rat bone marrow stem cells against hydrogen peroxide-induced cell death through antioxidation and activation of PI3K-Akt pathway. Neurosci Lett. 2012;516:247-52.

70. Chen Y, Cao CP, Li CR, Wang W, Zhang D, Han LL, et al. Ghrelin modulates insulin sensitivity and tau phosphorylation in high glucose-induced hippocampal neurons. Biol Pharm Bull. 2010;33:1165-9.

71. ZZ C, YP F. DL-3-n-butylphthalide attenuates reperfusion-induced blood-brain barrier damage after focal cerebral ischemia in rats. Zhongguo Yao Li Xue Bao. 1999;20:696-700.

72. Wu FF, Xu K, Xu KB, Teng CH, Zhang M, Xia LL, et al. DL-3n-butylphthalide improves traumatic brain injury recovery via inhibiting autophagy-induced blood-brain barrier disruption and cell apoptosis. J Cell Mol Med. 2020;24: 1220-32. 